\title{
Integrative analysis of long non-coding RNA and messenger RNA expression in toll-like receptor 4-primed mesenchymal stem cells of ankylosing spondylitis
}

\author{
Yu-Xi Li ${ }^{1 \#}$, Ting Liu ${ }^{2 \#}$, Yu-Wei Liang ${ }^{1}$, Jia-Jun Huang ${ }^{1}$, Jun-Shen Huang ${ }^{1}$, Xiang-Ge Liu ${ }^{1}$, \\ Zi-Ying Cheng ${ }^{1}$, Shi-Xin Lu ${ }^{1}$, Ming Li $^{1}$, Lin Huang ${ }^{1}$ \\ ${ }^{1}$ Department of Orthopaedics, Sun Yat-sen Memorial Hospital, Sun Yat-sen University, Guangzhou, China; ${ }^{2}$ Department of Anaesthesia, Sun Yat-sen \\ Memorial Hospital, Sun Yat-sen University, Guangzhou, China \\ Contributions: (I) Conception and design: YX Li; (II) Administrative support: YW Liang, JJ Huang, JS Huang; (III) Provision of study materials \\ or patients: M Li, L Huang; (IV) Collection and assembly of data: XG Liu, ZY Cheng, SX Lu; (V) Data analysis and interpretation: T Liu; (VI) \\ Manuscript writing: All authors; (VII) Final approval of manuscript: All authors. \\ \#These authors contributed equally to this work. \\ Correspondence to: Ming Li; Lin Huang. Department of Orthopaedics, Sun Yat-sen Memorial Hospital, Sun Yat-sen University, Guangzhou 510120, \\ China. Email: liming76@mail.sysu.edu.cn; huang15@mail.sysu.edu.cn.
}

Background: The precise pathogenesis of ankylosing spondylitis (AS) is still largely unknown at present. Our previous study found that toll-like receptor 4 (TLR4) downregulated and performed immunoregulatory dysfunction in mesenchymal stem cells from AS patients (AS-MSCs). The aim of this study was to explore the expression profiles of long non-coding RNAs (lncRNAs) and messenger RNAs (mRNAs) in TLR4primed AS-MSCs, and to clarify the potential mechanisms.

Methods: The immunoregulatory effects of MSCs were determined after TLR4 activation. Next, the differentially-expressed (DE) lncRNAs and mRNAs between AS-MSCs and TLR4-primed AS-MSCs [stimulated by lipopolysaccharide (LPS)] were identified via high-throughput sequencing followed by quantitative real-time PCR (qRT-PCR) confirmation. Finally, bioinformatics analyses were performed to identify the critical biological functions, signaling pathways, and associated functional networks involved in the TLR4-primed immunoregulatory function of AS-MSCs.

Results: A total of 147 DE lncRNAs and 698 DE mRNAs were identified between TLR4-primed ASMSCs and unstimulated AS-MSCs. Of these, 107 lncRNAs were upregulated and 40 were downregulated (fold change $\geq 2, \mathrm{P}<0.05$ ), while 504 mRNAs were upregulated and 194 were downregulated (fold change $\geq 2, \mathrm{P}<0.05$ ). Five lncRNAs and five $\mathrm{mRNAs}$ with the largest fold changes were respectively verified by qRTPCR. Gene ontology (GO) and Kyoto Encyclopedia of Genes and Genomes (KEGG) analyses demonstrated that the DE mRNAs and lncRNAs were highly associated with the inflammatory response, such as NODlike receptor (NLR) signaling pathway, the TNF signaling pathway and the NF- $\kappa$ B signaling pathway. Cisregulation prediction revealed eight novel lncRNAs, while trans-regulation prediction revealed 15 lncRNAs, respectively. Eight core pairs of lncRNA and target mRNA in the lncRNA-transcription factor (TF)-mRNA network were as follows: PACERR-PTGS2, LOC105378085-SOD2, LOC107986655-HIVEP2, MICB-DTMICB, LOC105373925-SP140L, LOC107984251-IFIT5, LOC112268267-GBP2, and LOC101926887IFIT3, respectively.

Conclusions: TLR4 activation in AS can enhance the immunoregulatory ability of MSCs. Eight core pairs of lncRNA and target mRNA were observed in TLR4-primed AS-MSCs, which could contribute to understanding the potential mechanism of AS-MSC immunoregulatory dysfunction.

Keywords: Ankylosing spondylitis (AS); mesenchymal stem cells (MSCs); toll-like receptor 4 (TLR4); long noncoding RNA (lncRNA); immunoregulation 
Submitted Aug 25, 2021. Accepted for publication Oct 16, 2021.

doi: 10.21037/atm-21-5020

View this article at: https://dx.doi.org/10.21037/atm-21-5020

\section{Introduction}

Ankylosing spondylitis (AS) is a chronic inflammatory rheumatic disease characterized by inflammatory back pain and asymmetrical peripheral arthritis (1). Previous studies have shown that AS is closely related to immune dysfunction; however, the pathogenesis of AS remains largely unknown. Mesenchymal stem cells (MSCs) are a group of self-renewing cells that have a significant immunomodulatory ability, which allows them to regulate $\mathrm{T}$ cell proliferation and differentiation and inhibit dendritic cell (DC) maturation (2,3). According to recent studies, abnormal immunoregulation by MSCs can lead to several autoimmune diseases, such as immune thrombocytopenia and systemic lupus erythematosus (SLE) (4-6). In our previous study, we isolated MSCs from the bone marrow (BM) aspirates of AS patients by density gradient centrifugation as described in our previous study (7). We found that reduced immunoregulatory ability of MSCs in AS patients may play a significant role in the pathogenesis of this disease (8). Furthermore, our clinical trial demonstrated that intravenous infusion of MSCs from healthy donors (HDs) was a feasible, safe, and effective approach for the treatment of AS (7). Our research results indicate that it is essential to elucidate the mechanism of abnormal immunoregulatory function of AS-MSCs. However, our understanding of the immunoregulatory function of ASMSCs is still in its infancy, and further characterization and identification of key factors regulating these properties are needed.

Toll-like receptors (TLRs), the most characterized pattern recognition receptors (PRRs), which are capable of potently activating different cell types, could be highly expressed on most immune cells, as well as other cell types including chondrocytes, endothelial cells, and fibroblasts (9). Their downstream signaling pathways lead to the production of a wide range of immune stimulatory cytokines and chemokines (10). Aberrant activation of TLRs may result in unrestricted inflammatory responses, suggesting that the TLR family may play a pivotal role in the development of autoimmune diseases (11). Additionally, TLR pathway activation can also generate autoimmunity as well as long-term inflammation (12). Emerging evidence further suggests a role for TLR in the pathogenesis of spondyloarthropathies, including AS (13). TLRs, especially TLR4, have also been demonstrated to play important roles in regulating the immunomodulatory properties of MSCs (14). Liotta et al. found that ligation of TLR4 suppressed the inhibitory effects of human BMMSCs on T cell proliferation by downregulating Jagged-1 expression (15). Waterman et al. demonstrated that MSCs could be primed towards a pro-inflammatory phenotype after TLR4 activation (16). In contrast, Opitz et al. reported that TLR4 enhanced the immunosuppressive properties of human BM-MSCs by directly inducing indoleamine 2,3-dioxygenase 1 (IDO1) (17). The expression of TLR4 is essential for the suppressive activity of MSCs; it has been demonstrated that TLR4 deletion is associated with a diminished suppressive effect in vitro and a loss of therapeutic effect in an experimental autoimmune encephalomyelitis (EAE) model in MSCs-TLR4 knockout mice (18). Our previous study found that the expression of TLR4 in MSCs was downregulated in AS patients compared to HDs (19). Hence, the reduced inhibitory effects of ASMSCs may be caused by the abnormal expression and function of TLR4. Unfortunately, inconsistent results have been reported in recent years, and the potential immunoregulatory mechanisms by which TLR4 controls the immunoregulatory ability of AS-MSCs remains unclear and must be addressed.

Non-coding RNAs (ncRNAs) are a class of non-proteincoding RNA and functional RNA molecules that include microRNA (miRNA), long non-coding RNAs (lncRNA), and circular RNAs (circRNA) (20). Overwhelming evidence has indicated that various ncRNAs are implicated in human disease processes. For example, miRNAs are key molecules in various diseases (21). In recent years, lncRNAs (>200 nt RNA molecules) have been found and studied to play a role in the development and evolution of disease. LncRNAs are important epigenetic regulators and thus participate in crucial roles in various cell biological behaviors (22). For example, lncRNA-PCAT1 negatively regulates miR-145-5p, which promotes TLR4 expression to stimulate osteogenic differentiation by activating the TLR signaling pathway in human adipose-derived stem cells (23). Specifically, lncRNAs are widely involved in the regulation of immune system homeostasis. LncRNA NEAT1 promotes the 
inflammatory response in sepsis-induced liver injury via the Let-7a/TLR4 axis (24). Lipopolysaccharide (LPS)induced lncRNA Mirt2 can function as a checkpoint to prevent aberrant activation of inflammation and is a potential regulator of macrophage polarization (25). LncRNA MALAT1 regulates inflammatory cytokine production in LPS-stimulated human gingival fibroblasts through sponging miR-20a and activating the TLR4 pathway (26). Though several key DE mRNAs and DE IncRNAs have been identified to try to provide some information for understanding the pathogenesis of AS and developing potential biomarkers for AS (27). However, the molecular mechanism by which IncRNAs participate in the immunoregulatory function of MSCs from AS, particularly after TLR4 activation by ligands stimulation, is still unclear.

The current study presents an integrative analysis of lncRNA-messenger RNA (mRNA) expression profiles and functional networks involved in the TLR4-primed immunoregulation of AS-MSCs. These results improve our understanding of the roles of lncRNAs in the immunoregulatory ability of AS-MSCs and could provide potential targets to improve the curative effect of MSCs in AS.

We present the following article in accordance with the MDAR reporting checklist (available at https://dx.doi. org/10.21037/atm-21-5020).

\section{Methods}

\section{AS-MSC isolation and cell culture}

The study was conducted in accordance with the Declaration of Helsinki (as revised in 2013). The present study was approved by the ethics committee of Sun Yatsen Memorial Hospital, Sun Yat-sen University (No. 2020KY-083). In addition, informed consent was obtained from all patients. MSCs were isolated from BM aspirates collected from AS patients who provided informed consent through density gradient centrifugation, as described in our previous study (7). After density gradient centrifugation, MSCs were isolated through plastic adherence, and grown at $37{ }^{\circ} \mathrm{C}$ in an atmosphere of $5 \% \mathrm{CO}_{2}$ for 1 week. The MSCs were trypsinized when the cultures reached 80-90\% confluence. MSCs in passages three to five were used in subsequent experiments. MSCs were identified on the basis of immunological phenotypes and the triple-lineage differentiation capability, as previously described (7). After identifying MSC immunophenotypic markers by flow cytometry, cells in passages three to five were used for subsequent experiments.

\section{Pre-stimulation of TLR4 on AS-MSCs}

To stimulate TLR4 on AS-MSCs effectively, determining the proper stimulating concentration and treatment time of the LPS (TLR4 ligand) was necessary. For the timebased stimulation test, $1.0 \mu \mathrm{g} / \mathrm{mL}$ LPS (Sigma-Aldrich, USA) was added to the culture medium at different times $(0,2,4,8,12$, and 24 hours) before a mixed lymphocyte reaction (MLR) was performed. For the concentrationbased stimulation test, different concentrations $(0,0.1,1$, and $10 \mu \mathrm{g} / \mathrm{mL}$ ) of LPS were added to cultured cells for the previously selected time. The best conditions for TLR4 activation in AS-MSCs were evaluated by assessing the level of p38 phosphorylation through western blotting. After determining the best stimulation time and concentration of LPS, MSCs were pre-primed before co-culturing with peripheral blood mononuclear cells (PBMCs) and gene analysis.

\section{Co-culture of AS-MSCs and PBMCs for PBMC proliferation analysis}

AS-MSCs were primed with $1 \mu \mathrm{g} / \mathrm{mL}$ LPS for 4 hours prior to co-culturing with PBMCs to activate TLR4. PBMCs were harvested from blood samples collected from HDs using the Ficoll-Paque density gradient centrifugation method and then labeled with carboxyfluorescein diacetate succinimidyl ester (CFSE; BD Bioscience, USA). PBMCs were incubated with $5 \mu \mathrm{M}$ CFSE in phosphate-buffered saline (PBS) at $37^{\circ} \mathrm{C}$ for 5 mins. Following incubation, the staining reaction was terminated by washing the PBMCs with complete medium twice. For co-culturing with AS-MSCs (effector cells), AS-MSCs at a density of $1 \times 10^{5}$ cells/well seeded in six-well plates were stimulated with or without $1 \mu \mathrm{g} / \mathrm{mL}$ LPS for 4 hours and then subjected to Co-60 irradiation (30 Gy). CFSE-labeled PBMCs (responder cells) at a cell density of $1 \times 10^{6}$ were then added to the MSC cultures. The co-cultures were stimulated with human anti-CD3/CD28 monoclonal antibodies (mAbs; CD3: $200 \mathrm{ng} / \mathrm{mL}$; CD28: $1 \mu \mathrm{g} / \mathrm{mL}$, BD Bioscience, USA) for 5 days, after which the PBMCs were harvested and stained with an anti-CD4-FITC antibody (BD Bioscience, USA). Cell proliferation was evaluated using flow cytometry. A minimum of 10,000 live cell events gated in scatter plots were analyzed for each sample. 


\section{Library construction and bigh-throughput sequencing}

Three MSC samples from five AS patients were selected randomly and separated into two subgroups: MSCs cultured without stimulation (control group, samples C1-C3) and MSCs stimulated with $1 \mu \mathrm{g} / \mathrm{mL}$ LPS for 4 hours to activate TLR4 specifically (experimental group, samples T1-T3). The total RNA concentration was quantified with a NanoDrop ND-2000 (Thermo Scientific, MA, USA), and RNA integrity was assessed using an Agilent Bioanalyzer 2100 (Agilent Technologies, USA). Sample labeling, microarray hybridization, and washing were performed according to the manufacturer's standard protocols. Briefly, total RNA was transcribed into doublestranded complementary DNA (cDNA), which was then synthesized into complementary RNA (cRNA) and labeled with Cyanine-3-CTP. The labeled cRNAs were hybridized onto the microarray. After washing, the arrays were scanned using an Agilent Scanner G2505C (Agilent Technologies, USA). The Agilent Human lncRNA Microarray 2018 (4×180 k, Design ID: 085630) was used in this experiment, and data analysis of the six samples was conducted by $\mathrm{OE}$ Biotechnology Co., Ltd. (Shanghai, China).

\section{Expression analysis}

Feature Extraction software (version 10.7.1.1, Agilent Technologies) was used to analyze array images to obtain raw data. GeneSpring (version 14.8, Agilent Technologies) was employed to complete the basic analysis with the raw data. First, the raw data were normalized with the quantile algorithm. Probes with at least one condition out of two conditions having flags in "Detected" were chosen for further data analysis. Differentially-expressed (DE) genes were then identified through fold change data as well as the $\mathrm{P}$ value calculated with a $t$-test. The threshold for up- and downregulated genes was set at a fold change $\geq 2.0$ and a $\mathrm{P}$ value $\leq 0.05$. Afterwards, gene ontology (GO) and Kyoto Encyclopedia of Genes and Genomes (KEGG) analyses were applied to determine the roles of these DE mRNAs. Finally, hierarchical clustering was performed to display the distinguishable gene expression patterns among samples.

\section{Quantitative real-time PCR ( $q R T-P C R)$ validation}

To validate the reliability of high-throughput RNAsequencing (RNA-seq) and explore the expression trends of mRNAs and lncRNAs, we performed qRT-PCR for biological validation. Total RNA was isolated from AS-
MSCs with or without LPS stimulation using TRIzol reagent (Thermo Scientific, MA, USA) according to the manufacturer's protocol. cDNA was transcribed using a PrimeScript RT reagent kit (Takara, Otsu). QRT-PCR was then performed, and the data were analyzed using the $2^{-\Delta \Delta C t}$ method. The primer sequences used in the qRT-PCR assay are provided in Table S1.

\section{Co-expression network of lncRNAs with mRNAs}

To further examine the potential roles of DE lncRNAs and mRNAs in TLR4-primed AS-MSCs, a DE lncRNA-mRNA co-expression network was constructed. First, the Pearson correlation coefficients (PCC) between the expression levels of each DE IncRNA-DE mRNA pair in the TLR4-primed AS-MSCs and unstimulated AS-MSCs were calculated. Next, the DE lncRNA-DE mRNA pairs with an absolute PCC value $\geq 0.85$ and $\mathrm{P}<0.05$ were defined as co-expressed DE lncRNA-DE mRNA pairs. The co-expressed DE IncRNA-DE mRNA pairs in which the expression level of the DE mRNA was positively correlated with the expression level of the DE lncRNA in TLR4-primed AS-MSCs were defined as positively co-expressed DE IncRNA-DE mRNA pairs. In contrast, the co-expressed DE IncRNA-DE mRNA pairs in which the expression level of the DE mRNA was negatively correlated with that of the DE lncRNA in TLR4-primed AS-MSCs were defined as negatively coexpressed DE lncRNA-DE mRNA pairs. The co-expressed DE lncRNA-DE mRNA networks were visualized using Cytoscape 3.0 software (National Institute of General Medical Sciences, USA).

\section{Cis- and trans-regulation predictions of DE IncRNAs}

It has been suggested that lncRNAs regulate gene expression through both cis- and trans-regulation. For cisregulation prediction, we identified each paired lncRNA and mRNA using the following procedures: (I) the mRNA loci were within $100-\mathrm{kb}$ windows upstream or downstream of the given lncRNA; and (II) the Pearson correlation of lncRNA-mRNA expression was significant $(\mathrm{P} \leq 0.05)$. For trans-regulation prediction, we enriched the co-expressed mRNAs with DE lncRNAs that significantly overlapped with the host genes of transcription factors (TFs). Using the threshold $\mathrm{P}<0.05$, each IncRNA could be connected with one to more than a dozen TFs, and each pair of lncRNATF was the result of several gene enrichments based on the hypergeometric cumulative distribution function. We 
then constructed the lncRNA-TF-mRNA network using Cytoscape software.

\section{Statistical analysis}

Data were expressed as the mean \pm standard deviation (SD) and were analyzed using the statistical software package SPSS16.0 (IBM, USA). Pearson correlation was used for lncRNA-mRNA co-expression analyses. A $\mathrm{P}$ value $<0.05$, fold enrichment $>2$, and $\log _{2}$ fold change $>1$ were considered statistically significant.

\section{Results}

\section{The effect of TLR4 activation on MSCs is time- and dose- dependent}

To investigate whether the activation of TLR4 in MSCs can affect the immunoregulatory ability of these cells, MSCs from AS patients were pre-stimulated with LPS before being co-cultured with PBMCs. To determine the best stimulation time and concentration for the TLR4 ligand used, the level of p38 phosphorylation was examined by western blotting. AS-MSCs were first exposed to LPS at a concentration of $1 \mu \mathrm{g} / \mathrm{mL}$ for the indicated times $(0,2,4$, 8,12 , and 24 hours) and then treated with three different concentrations $(0,0.1,1$, and $10 \mu \mathrm{g} / \mathrm{mL})$ of stimuli for the previously selected time. The upregulation of the phospho-p38 level was highest at 4 hours with a LPS concentration of $1 \mu \mathrm{g} / \mathrm{mL}$, and declined thereafter (Figure 1A).

\section{TLR4-primed AS-MSCs demonstrate an enhanced inbibitory effect on $\mathrm{CD}^{+} \mathrm{T}$ cell proliferation}

Previous reports have shown that a co-culture of unprimed MSCs with PBMCs can inhibit the proliferation and/ or activation of PBMCs (28). Thus, we sought to assess the potential influence of TLR 4 activation on the immunoregulatory effect of MSCs derived from AS patients. To this end, MSC-PBMC co-culturing was conducted with CFSE-labeled PBMCs (responder cells) co-cultured with unprimed or TLR4-primed MSCs (effector cells) for 5 days, after which time the proliferating responders were sorted by flow cytometry for CD4 positivity and then gated on CFSE expression. As shown in Figure $1 B, 72.8 \%$ of the $\mathrm{CD}^{+} \mathrm{T}$ cells underwent proliferation when cultured without MSCs, which was reduced to $46.3 \%$ when cultured with ASMSCs. Activation of TLR4 with $1 \mu \mathrm{g} / \mathrm{mL}$ LPS significantly enhanced the immunoregulatory effect of AS-MSCs, which reduced $\mathrm{CD}^{+} \mathrm{T}$ cell proliferation to $40.3 \%(\mathrm{P}<0.05)$ (Figure $1 B$ ). qRT-PCR results suggested that the expression of several cytokines and chemokines (TNF- $\alpha, C X C L 9$, $P D L 1, I L-1 \beta, I L-6$, and $i N O S)$ was strengthened after stimulation with LPS (Figure 1C).

\section{Identification of DE mRNAs and lncRNAs}

A total of 698 mRNAs were DE in TLR4-primed MSCs compared to unprimed MSCs from AS patients. Among these genes, 594 mRNAs were upregulated and 104 mRNAs were downregulated. The DE mRNAs are depicted using a clustergram (Figure 2A). The 20 mRNAs with the largest fold changes are shown in Table 1. Several immunoregulatory cytokines and chemokines, including CXCL10, CXCL11, IDO1, CXCL8, CXCL1, CCL20, IL-6, and SOD2, which are secreted by MSCs and play important roles in regulating immunocytes, were included in this list. A total of 147 lncRNAs, including 107 upregulated and 40 downregulated lncRNAs, were DE in TLR4-primed MSCs compared to unprimed MSCs from AS patients. The DE lncRNAs are depicted using a clustergram (Figure 2B). Both of the DE mRNA and LncRNAs are also showed by volcano plots (Figure 2C,2D). The 10 lncRNAs with the largest fold changes are shown in Table 2.

\section{Validation of DE mRNA and lncRNA expression levels}

To confirm the RNA-seq results, several important DE mRNAs and lncRNAs were assessed by qRT-PCR. We found that the expression of mRNAs (CXCL1, CXCL8, CXCL10, CXCL11, and CCL20) and IncRNAs (MIR3142HG, LOC105371619, LOC105374444, PACERR, and $L O C 105375914$ ) was significantly upregulated in TLR4-primed AS-MSCs compared to unstimulated ASMSCs $(\mathrm{P}<0.05)$ (Figure $3 A, 3 B)$. All qRT-PCR results were consistent with the RNA-seq results, confirming the reliability of the sequencing data.

\section{GO and KEGG analyses}

We performed GO analysis of the DE mRNAs and lncRNAs. The top $10 \mathrm{GO}$ terms related to biological processes, cellular components and molecular functions are provided in Figure $4 A$ and Table S2. In the biological processes category, the top five GO terms associated with DE mRNAs were defense response to virus, the type I interferon signaling pathway, the interferon-gamma- 
A
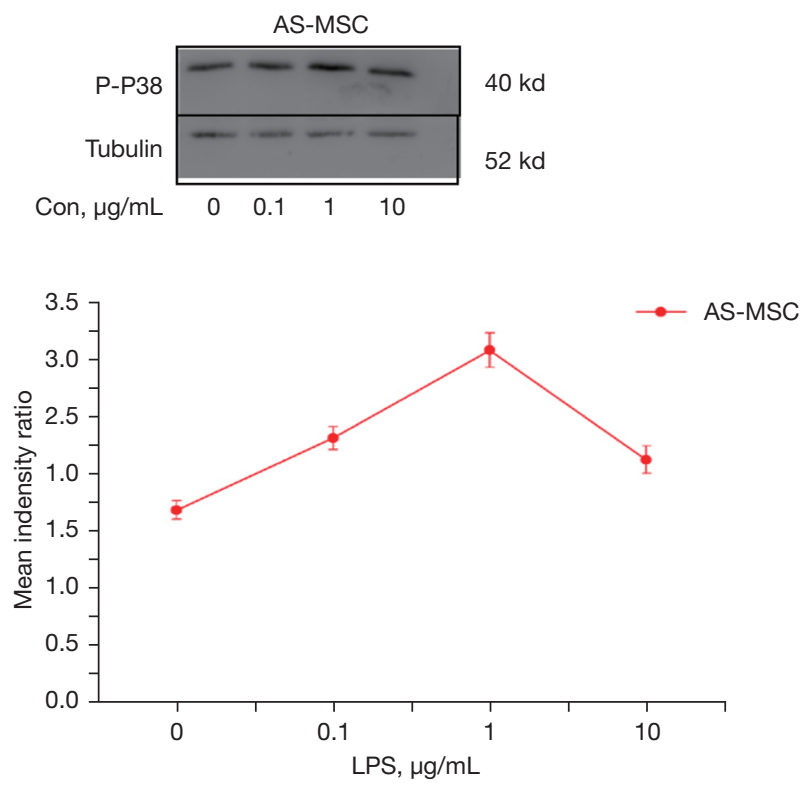

B
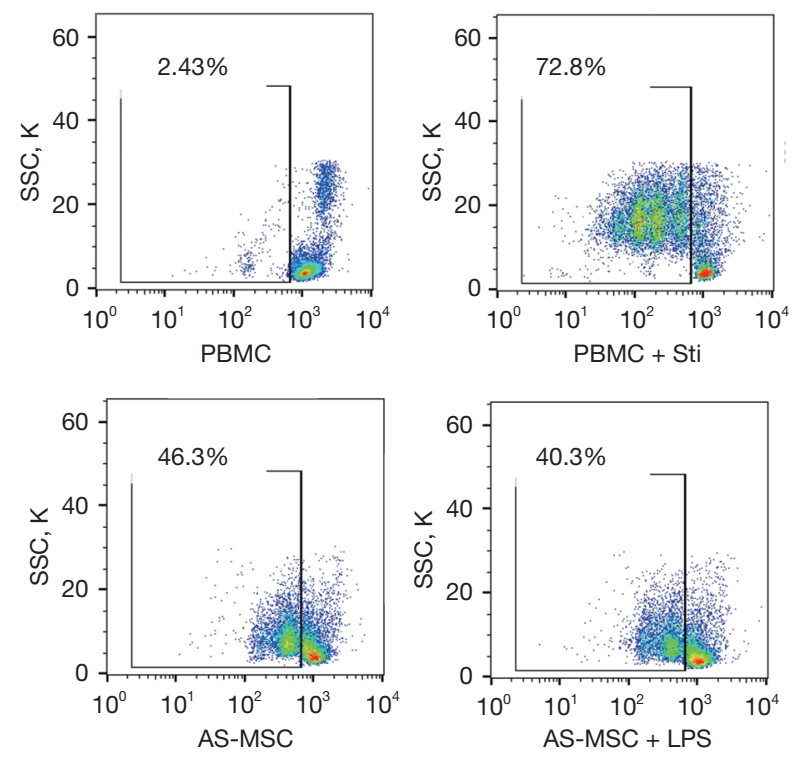
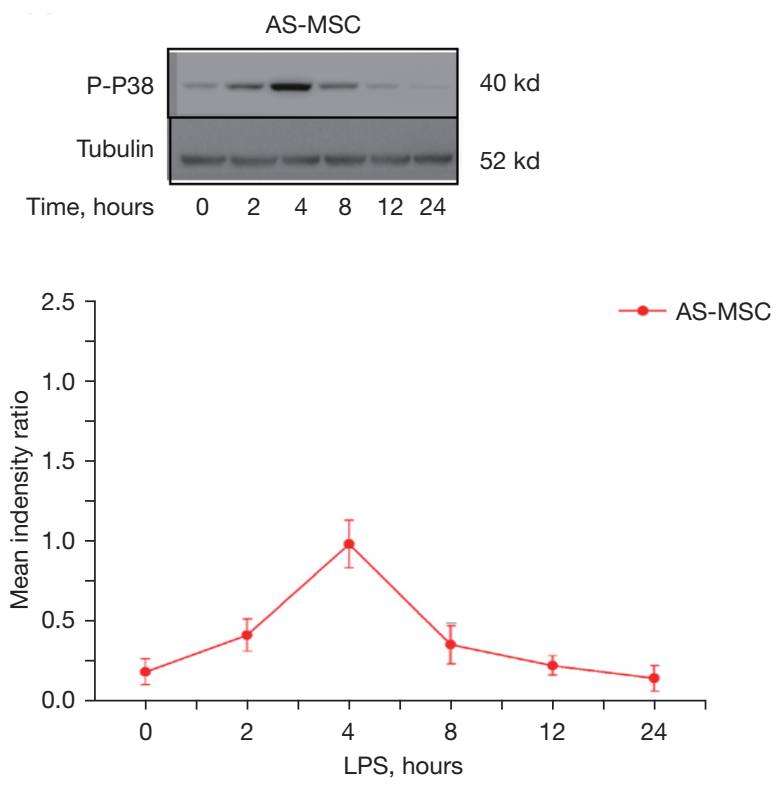

C

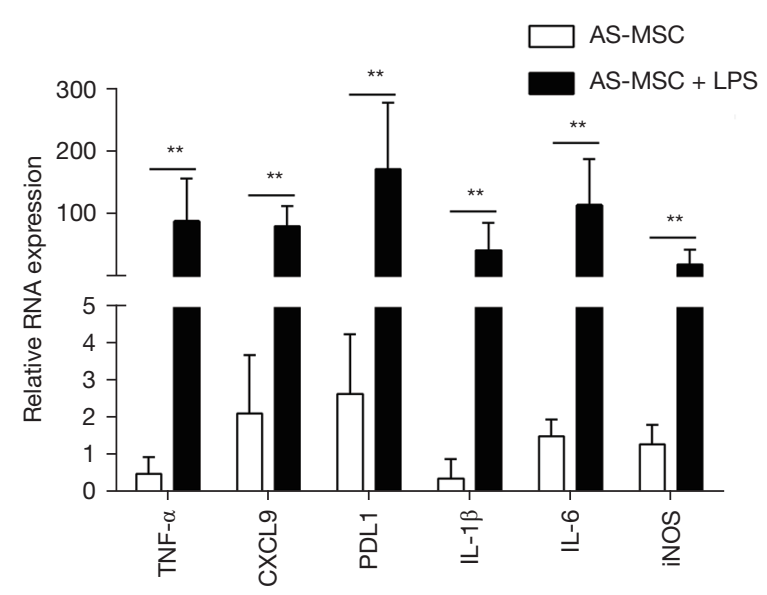

Figure 1 The immunoregulatory function of TLR4-primed AS-MSCs. (A) To determine the best duration and concentration of TLR4 ligand pre-stimulation, we examined the level of $\mathrm{p} 38$ phosphorylation by western blotting. AS-MSCs were exposed to LPS at four different concentrations $(0,0.1,1$, and $10 \mu \mathrm{g} / \mathrm{mL})$ for 4 hours or treated at a concentration of $1 \mu \mathrm{g} / \mathrm{mL}$ for the indicated time $(0,2,4,8,12$, or 24 hours). The upregulation of the phospho-p38 level was highest at 4 hours with a concentration of $1 \mu \mathrm{g} / \mathrm{mL}$ LPS, and declined thereafter. (B) AS-MSCs were pre-stimulated with or without $1 \mu \mathrm{g} / \mathrm{mL}$ LPS for 4 hours and then co-cultured with PBMCs at a ratio of 1:10 (MSCs: PBMCs) for 5 days. All PBMCs were then collected for assessment by flow cytometry to determine the positive percentage of CFSE-diluted cells (gated) to evaluate proliferation. AS-MSCs inhibited the proliferation of PBMCs, and this effect was strengthened by the activation of TLR4. (C) The gene expression of cytokines and chemokines in AS-MSCs after LPS stimulation was detected by qRT-PCR. **, P<0.01. TLR4, toll-like receptor 4; AS, ankylosing spondylitis; MSCs, mesenchymal stem cells; LPS, lipopolysaccharide; PBMCs, peripheral blood mononuclear cells; CFSE, carboxyfluorescein diacetate succinimidyl ester. 
A

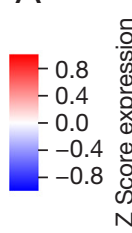

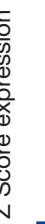

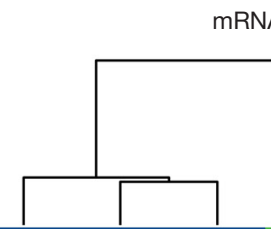

mRNA

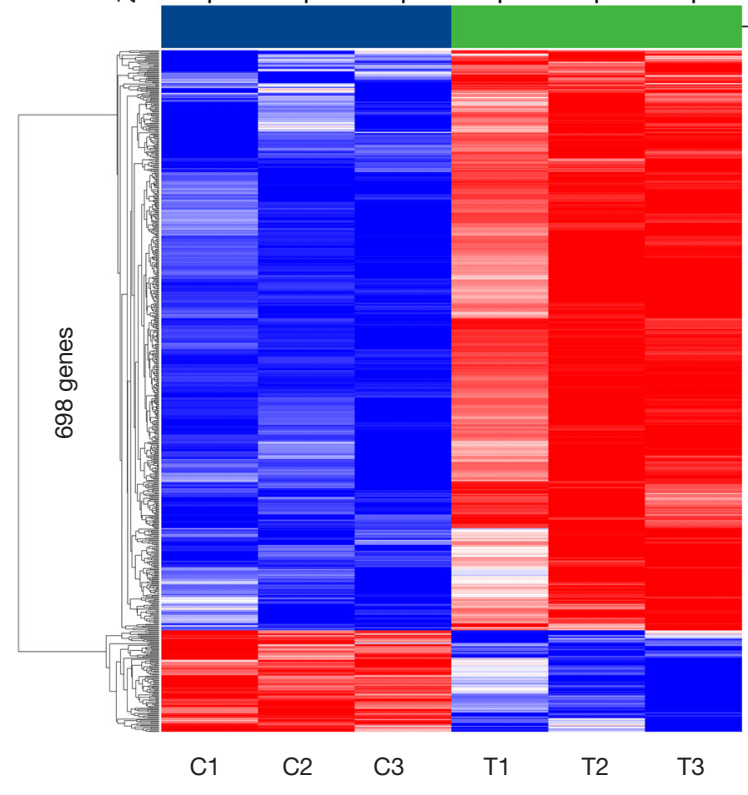

C

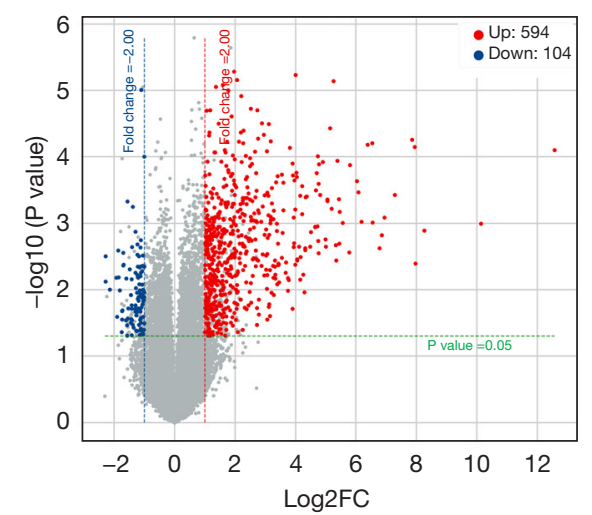

B
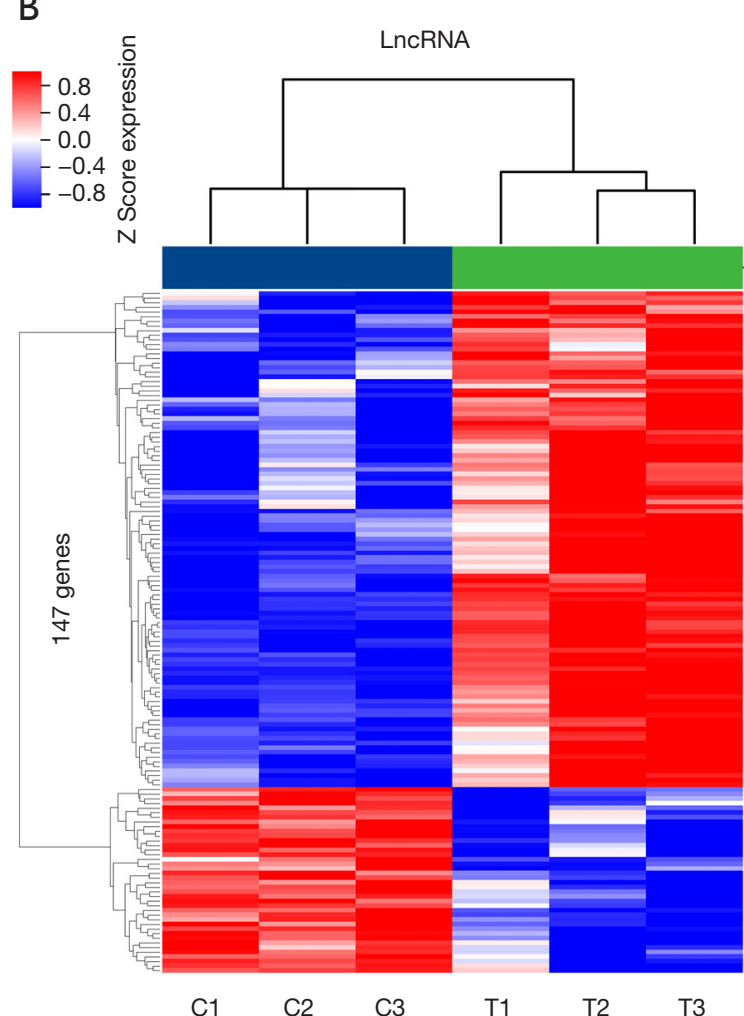

$\mathrm{D}$

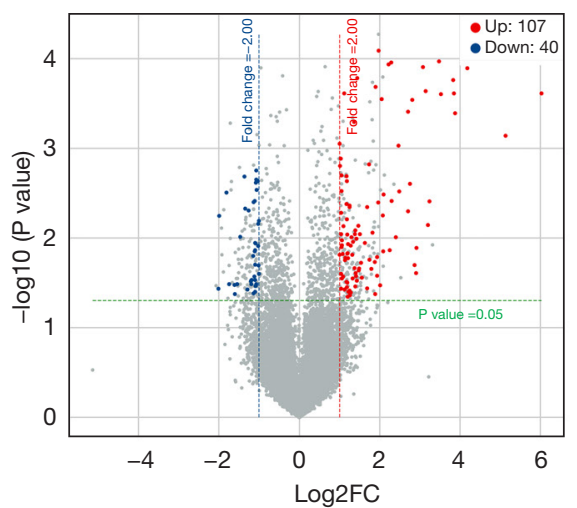

Figure 2 Identification of DE lncRNAs and mRNAs. (A) Heatmaps of DE mRNAs between TLR4-primed AS-MSCs and unstimulated AS-MSCs. (B) Heatmaps of DE lncRNAs between TLR4-primed AS-MSCs and unstimulated AS-MSCs. (C) Volcano plots of DE mRNAs between TLR4-primed AS-MSCs and unstimulated AS-MSCs. (D) Volcano plots of DE lncRNAs between TLR4-primed AS-MSCs and unstimulated AS-MSCs. DE, differentially-expressed; lncRNAs, long non-coding RNAs; mRNAs, messenger RNAs; TLR4, toll-like receptor 4; AS, ankylosing spondylitis; MSCs, mesenchymal stem cells; FC, fold change.

mediated signaling pathway, inflammatory response, and response to virus. In the molecular function category, the top five GO terms associated with the DE mRNAs were chemokine activity, CXCR chemokine receptor binding, ubiquitin-protein transferase activity, double-stranded RNA binding and TF activity, and sequence-specific DNA binding. In the cellular component category, the top five GO terms associated with the DE mRNAs were cytoplasm, 
Table 1 The characteristics of mRNAs with the largest fold change

\begin{tabular}{|c|c|c|c|}
\hline Gene name & Accession no. & $\log _{2}$ fold change & Regulation \\
\hline CXCL10 & NM_001565.3 & 12.58 & Up \\
\hline CXCL11 & NM_005409.4 & 10.14 & Up \\
\hline RSAD2 & NM_080657.4 & 8.27 & Up \\
\hline IDO1 & NM_002164.5 & 7.98 & Up \\
\hline CXCL8 & NM_000584.3 & 7.95 & Up \\
\hline CXCL1 & NR_046035.1 & 7.86 & Up \\
\hline IFIT1 & NM_001270930.1 & 7.30 & Up \\
\hline OASL & NM_003733.3 & 6.95 & Up \\
\hline CCL2O & NM_004591.2 & 6.86 & Up \\
\hline CXCL3 & NM_002090.2 & 6.79 & Up \\
\hline CCL5 & NM_001278736.1 & 6.56 & Up \\
\hline IFIT3 & NM_001289758.1 & 6.54 & Up \\
\hline CMPK2 & NM_207315.3 & 6.39 & Up \\
\hline$C D 274$ & NM_001267706.1 & 6.19 & Up \\
\hline OASL & NM_198213.2 & 6.08 & Up \\
\hline PTGS2 & NM_000963.3 & 6.04 & Up \\
\hline IL6 & NM_001318095.1 & 5.81 & Up \\
\hline SOD2 & NM_001322816.1 & 5.79 & Up \\
\hline CXCL8 & NM_001354840.1 & 5.56 & Up \\
\hline GBP5 & NM_052942.3 & 5.47 & Up \\
\hline
\end{tabular}

mRNAs, messenger RNAs.

cytosol, extracellular space, nucleus, and extracellular region.

KEGG analysis of the DE mRNAs determined that 75 pathways were significantly altered in TLR4-primed MSCs from AS patients. The top 30 affected pathways are shown in Figure 4B. The top 10 pathways and DE mRNAs associated with these pathways are shown in Table S3. The top pathways included the NOD-like receptor (NLR) signaling pathway, the TNF signaling pathway, the NF- $\mathrm{KB}$ signaling pathway, cytokine-cytokine receptor interaction, the IL-17 signaling pathway, and the TLR signaling pathway, which contribute to the immunoregulatory function of AS-MSCs.

\section{Interaction and co-expression network analyses}

The interactions between proteins encoded by DE mRNAs are shown in Figure 5A. OAS2, OAS3, OAS1, OASL, STAT1, IRF9, HLA-F, IRF1,IRF2, and HERC5 were identified as key genes that interact with many other DE mRNAs in this network. Based on the expression levels of DE lncRNAs and DE mRNAs, the PCC describing the co-expression association between 147 DE lncRNAs and 698 DE mRNAs was calculated. A total of 1,072 DE lncRNA-DE mRNA co-expression pairs were obtained, with an absolute PCC value $\geq 0.85$ and $\mathrm{P}<0.05$. Among these pairs, 706 lncRNAmRNA pairs were identified as being positively coexpressed, whereas 366 lncRNA-mRNA pairs were found to be negatively co-expressed (Figure $5 B$ ).

\section{Cis-regulation prediction of DE $\operatorname{lncRNAs}$}

Cis-regulation, which regulates the transcription of nearby genes located on the same chromosome, is vital for gene expression. A total of eight lncRNA transcripts and their predicted cis-regulated protein-coding genes were identified in the top 20 cis-regulated genes (Figure 6A). LOC107986655 was predicted to cis-regulate HIVEP2; $P A C E R R$ was predicted to cis-regulate PTGS2; MICB$D T$ was predicted to cis-regulate MICB; LOC105373925 was predicted to cis-regulate SP140L; LOC107984251 was predicted to cis-regulate IFIT5; LOC112268267 was predicted to cis-regulate $G B P 2 ; L O C 101926887$ was predicted to cis-regulate IFIT3; and LOC105378085 was predicted to cis-regulate $S O D 2$. These networks may provide valuable clues about these lncRNAs and their nearby coding genes in the development of AS.

\section{Trans-regulation prediction of DE IncRNAs}

One of the important mechanisms through which lncRNAs function is by participating in particular pathways regulated by TFs. In this study, a top 500 lncRNA-TF network was constructed to provide key data for subsequent research, and showed that 15 lncRNAs participated in pathways regulated by TFs (Figure $6 B$ ). We then selected the abovementioned relationships among the lncRNAs and TFs and further introduced the target mRNAs to build the lncRNA-TFmRNA network. Eight core pairs of lncRNAs and target mRNAs in the lncRNA-TF-mRNA network were obtained: PACERR-PTGS2, LOC105378085-SOD2, LOC107986655HIVEP2, MICB-DT-MICB, LOC105373925-SP140L, LOC107984251-IFIT5, LOC112268267-GBP2, and LOC101926887-IFIT3 (Figure 6C). 
Table 2 The characteristics of lncRNAs with the largest fold change

\begin{tabular}{|c|c|c|c|c|c|c|c|c|c|}
\hline Gene symbol & Accession no. & Fold change & Regulation & Chromosome & Strand & Start & End & Feature & Size (bp) \\
\hline LOC105370436 & XR_943719.2 & 35.08 & Up & 14 & + & 31271945 & 31276870 & ncRNA & 626 \\
\hline LOC105376014 & XR_929550.3 & 18.12 & Up & 9 & - & 32450235 & 32454362 & ncRNA & 2,133 \\
\hline MIR3142HG & NR_132748.1 & 14.70 & Up & 5 & + & 160468251 & 160487426 & ncRNA & 2,318 \\
\hline LOC105369543 & XR_948124.3 & 14.25 & Up & 11 & + & 123651728 & 123672257 & ncRNA & 3,095 \\
\hline LOC105374444 & XR_925284.2 & 11.54 & Up & 4 & + & 47627252 & 47636569 & ncRNA & 1,416 \\
\hline ANO7L1 & XR_946991.2 & 11.17 & Up & 1 & - & 16215438 & 16217420 & miscRNA & 1,466 \\
\hline PACERR & NR_125801.1 & 9.48 & Up & 1 & + & 186680654 & 186681446 & ncRNA & 793 \\
\hline
\end{tabular}

LncRNAs, long non-coding RNAs; miscRNA, miscellaneous RNA; ncRNAs, non-coding RNA.
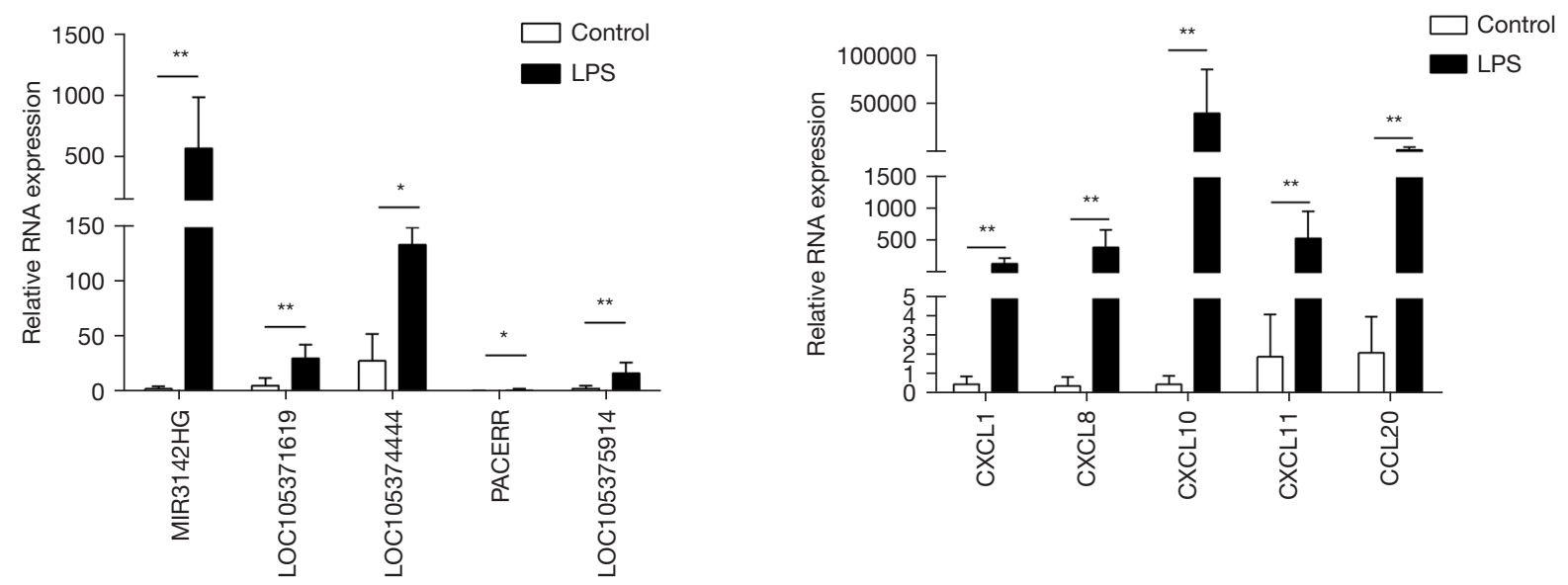

Figure 3 Validation of DE mRNAs and lncRNAs. (A) DE lncRNAs were confirmed by qRT-PCR. (B) DE mRNAs were confirmed by qRT-PCR. *, $\mathrm{P}<0.05$; **, $\mathrm{P}<0.01$. DE, differentially-expressed; mRNAs, messenger RNAs; lncRNAs, long non-coding RNAs; qRT-PCR, quantitative real-time PCR; LPS, lipopolysaccharide.

\section{Discussion}

In our present research, we utilized high-throughput sequencing followed by bioinformatics analysis to investigate the mRNA and lncRNA expression profiles and functional networks of TLR4-primed AS-MSCs. These findings were then confirmed by qRT-PCR. KEGG pathway analysis indicated that some key pathways, such as the NF$\kappa \mathrm{B}$ and TLR signaling pathways, might contribute to the immunoregulatory function of TLR4-primed AS-MSCs. In addition, we obtained novel findings via bioinformatics analyses of DE transcripts, including identification of the most significantly altered GO categories, construction of a co-expression network for lncRNA function prediction, and cis- and trans-regulation predictions of lncRNAs. Our results provide a model that can be used to explore the roles of lncRNAs and mRNAs in the immunoregulatory mechanisms of TLR4-primed AS-MSCs.

MSCs are among the most important immunoregulatory cell types, and regulate the functions of many immune cells, including T cells, B cells, and DCs (29-31). Abnormal immunoregulation by MSCs can lead to several autoimmune diseases (4). MSCs from AS patients cannot 
A

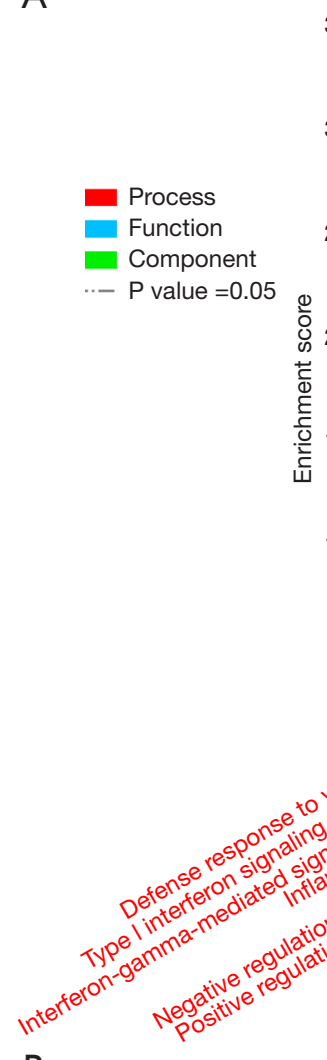

B

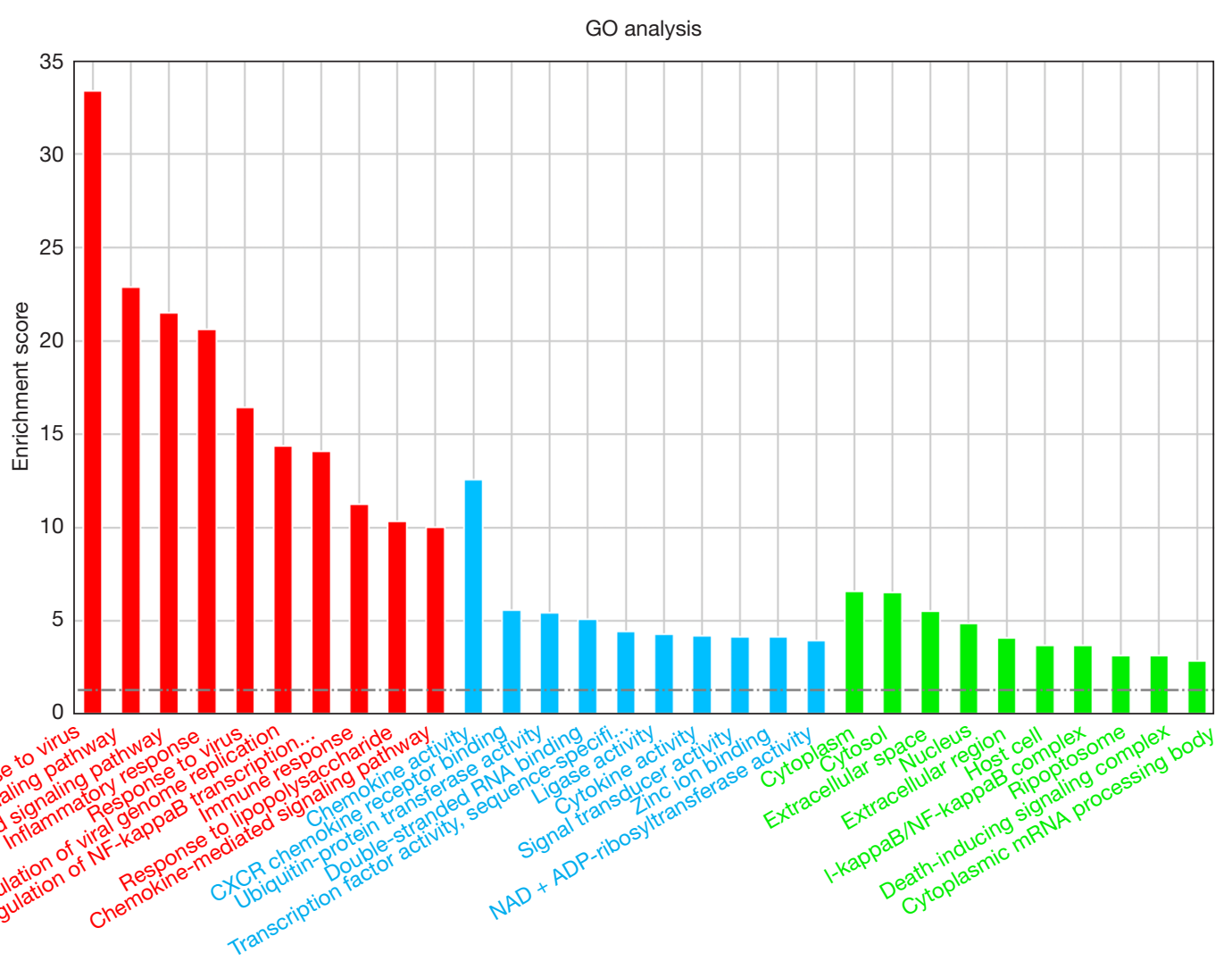

KEGG analysis

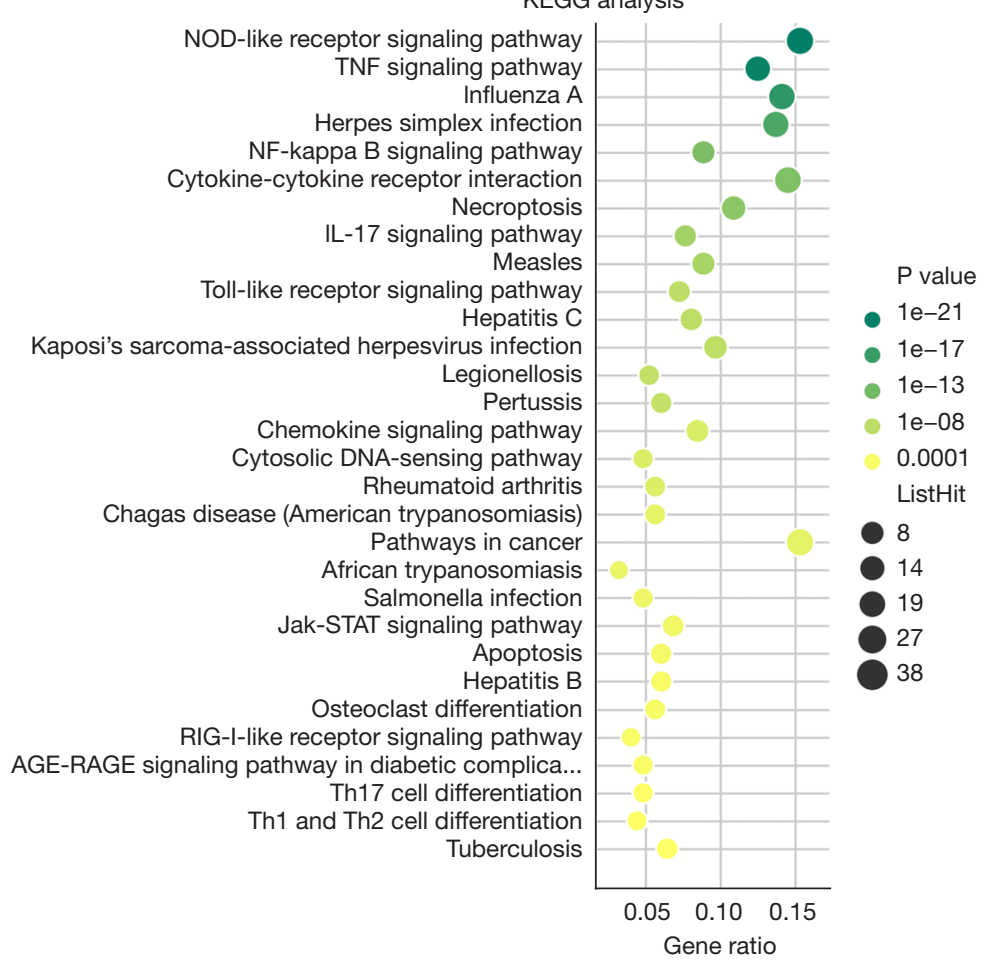

Figure 4 GO and KEGG analyses of DE mRNAs. (A) The top 10 terms identified by GO analysis of molecular functions, biological processes, and cellular components are shown. (B) DE mRNAs were clustered by KEGG analysis, and the top 30 pathways are shown. GO, gene ontology; KEGG, Kyoto Encyclopedia of Genes and Genomes; DE, differentially-expressed; mRNAs, messenger RNAs. 


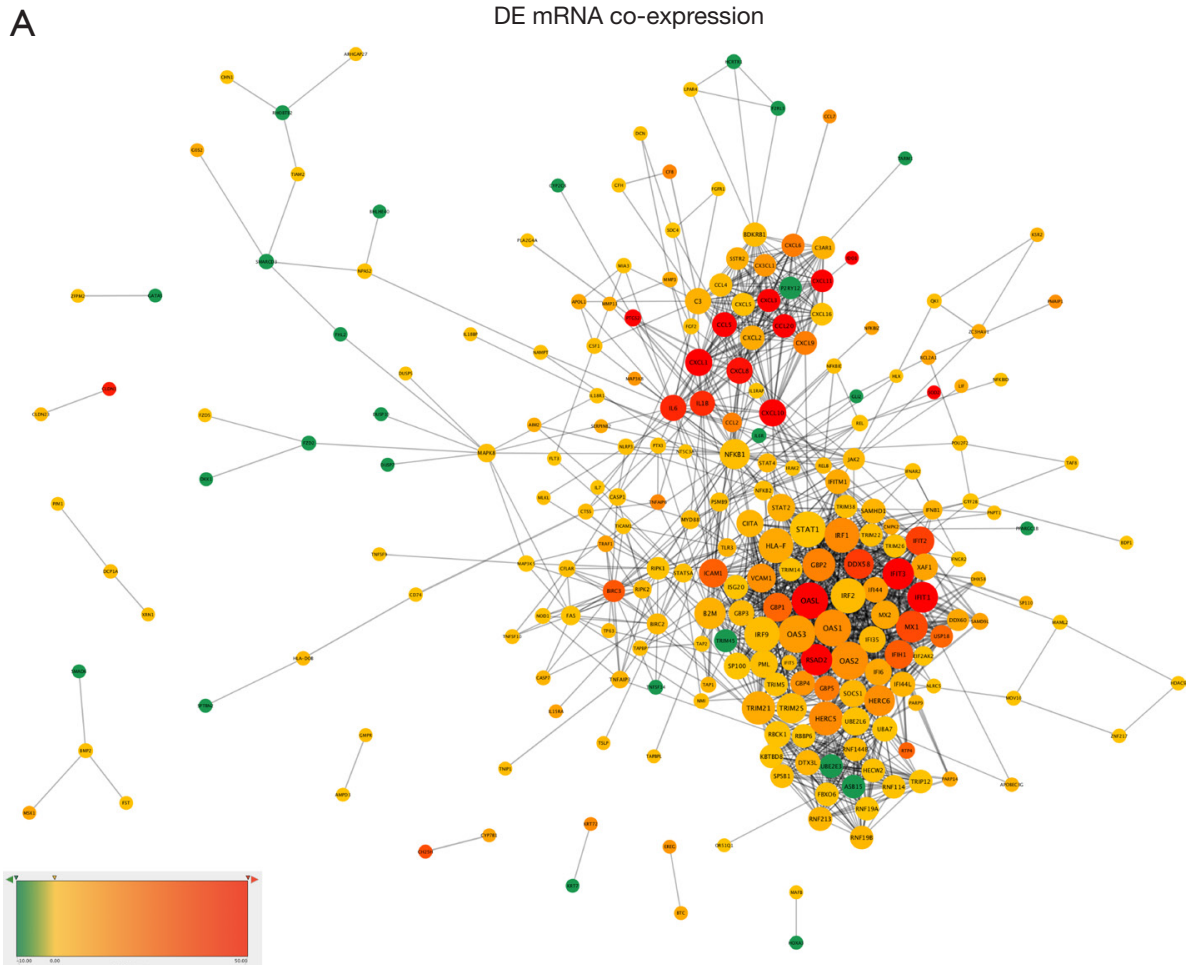

B

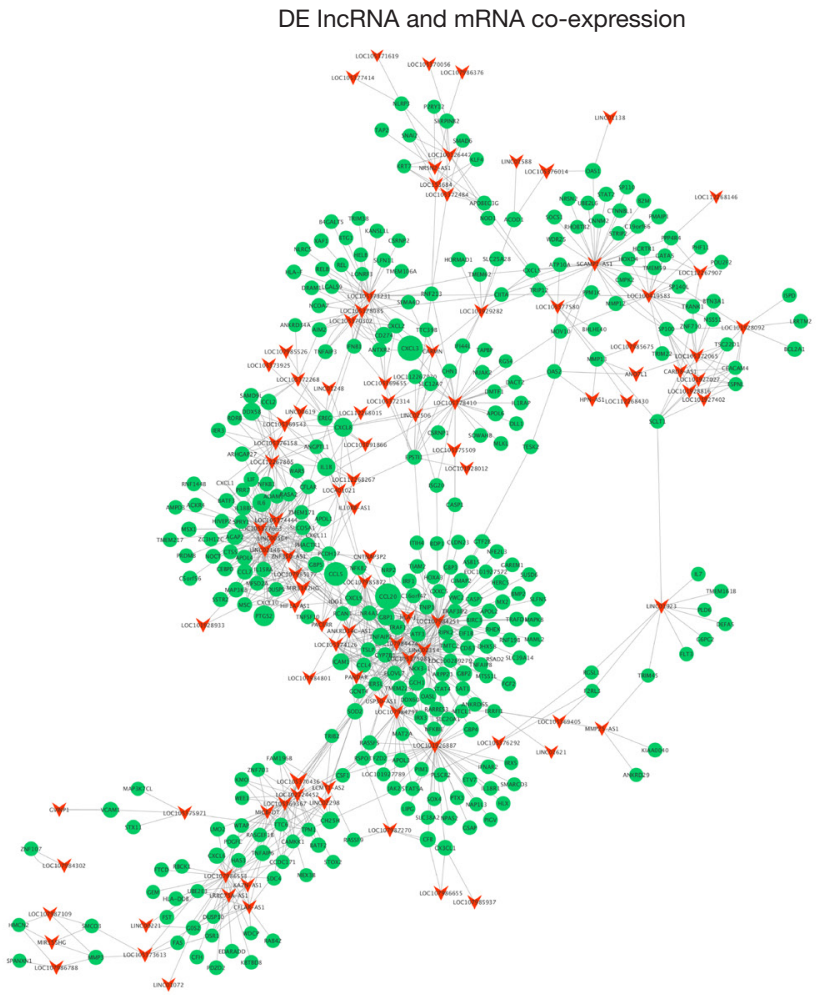

Figure 5 Advanced analyses of DE lncRNAs and mRNAs. (A) Interactions among mRNAs. Red and green represent upregulated and downregulated mRNAs, respectively. The size of the ellipse represents the fold change of DE mRNAs. (B) Co-expression network of DE lncRNAs and DE mRNAs. Triangle and ellipse represent lncRNA and mRNA, respectively. The size of the ellipse represents the fold change of DE mRNAs. DE, differentially-expressed; lncRNAs, long non-coding RNAs; mRNAs, messenger RNAs. 
A

Cis-regulation (Top 20)

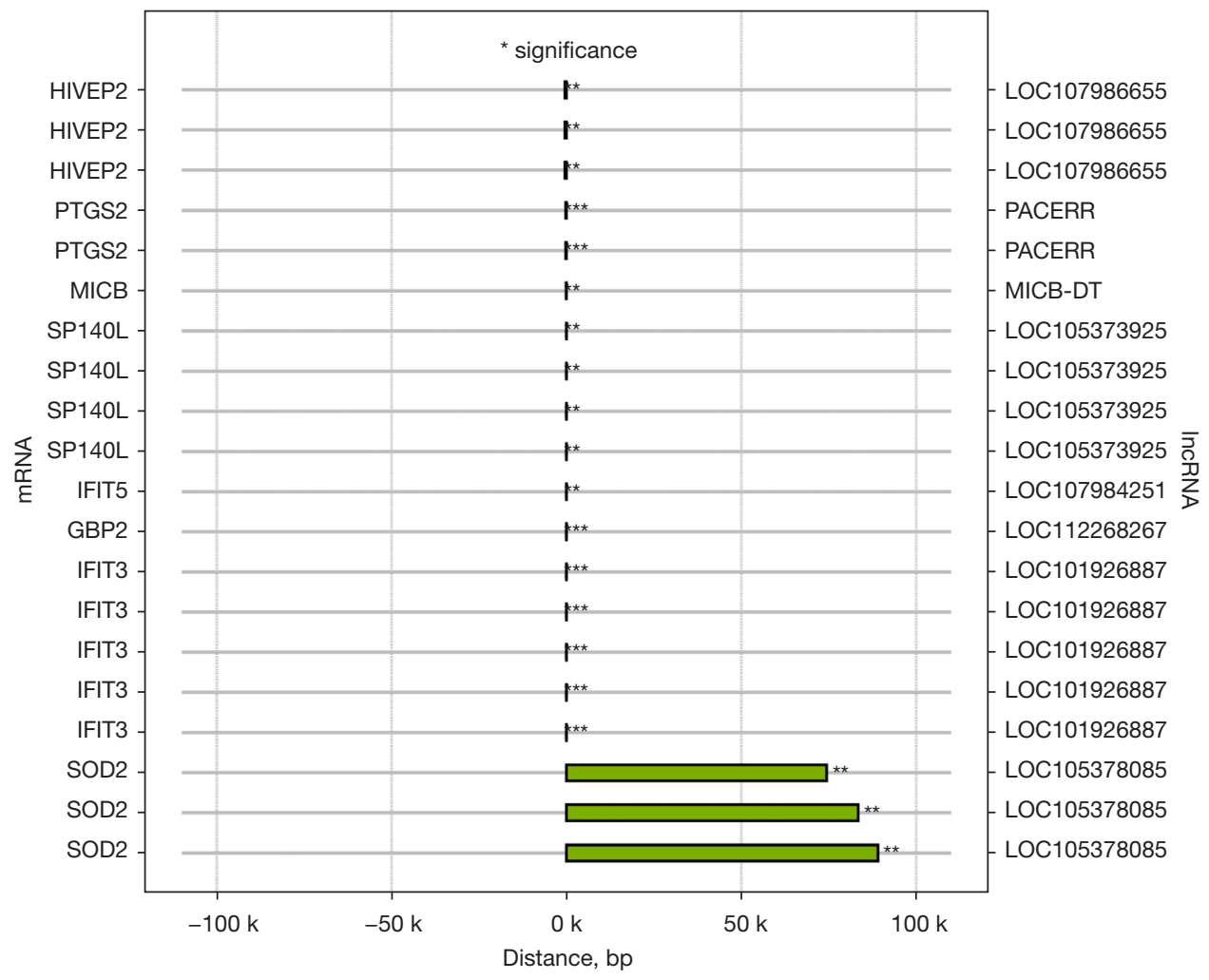

B

Trans-regulation (top 500)

LINOO1923

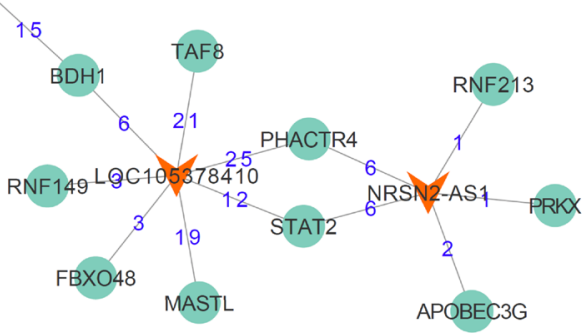

ZC3HAV1 USP42

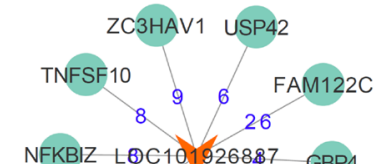

NFKBIZ LOC10) 226887 GBP4

RNF19B $84412^{51}$ IRF1

ZNFX1 PARP14

APOBEC $3 G$
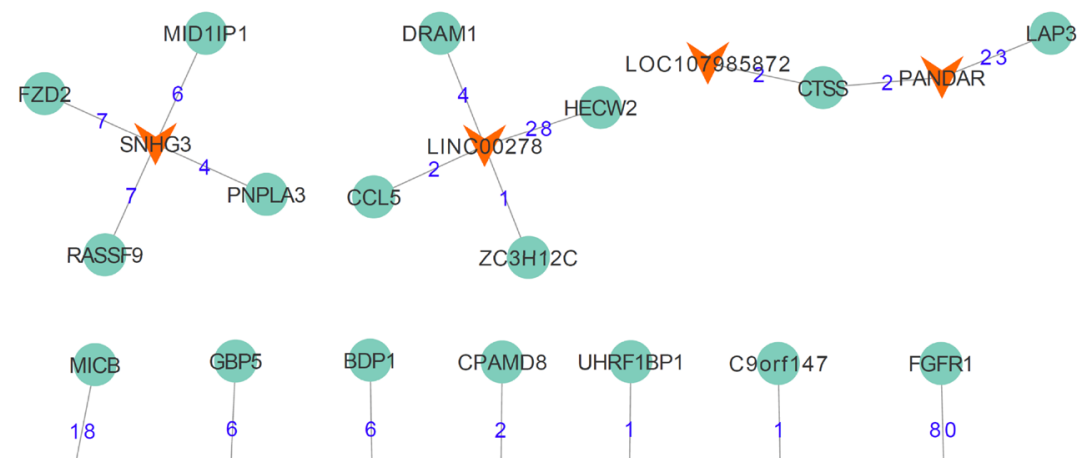

LOC105872268 LINCO06564 ZNF350-AS1 MIÖE-DT LINC00221 LINCO2298 LOC101928933

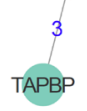




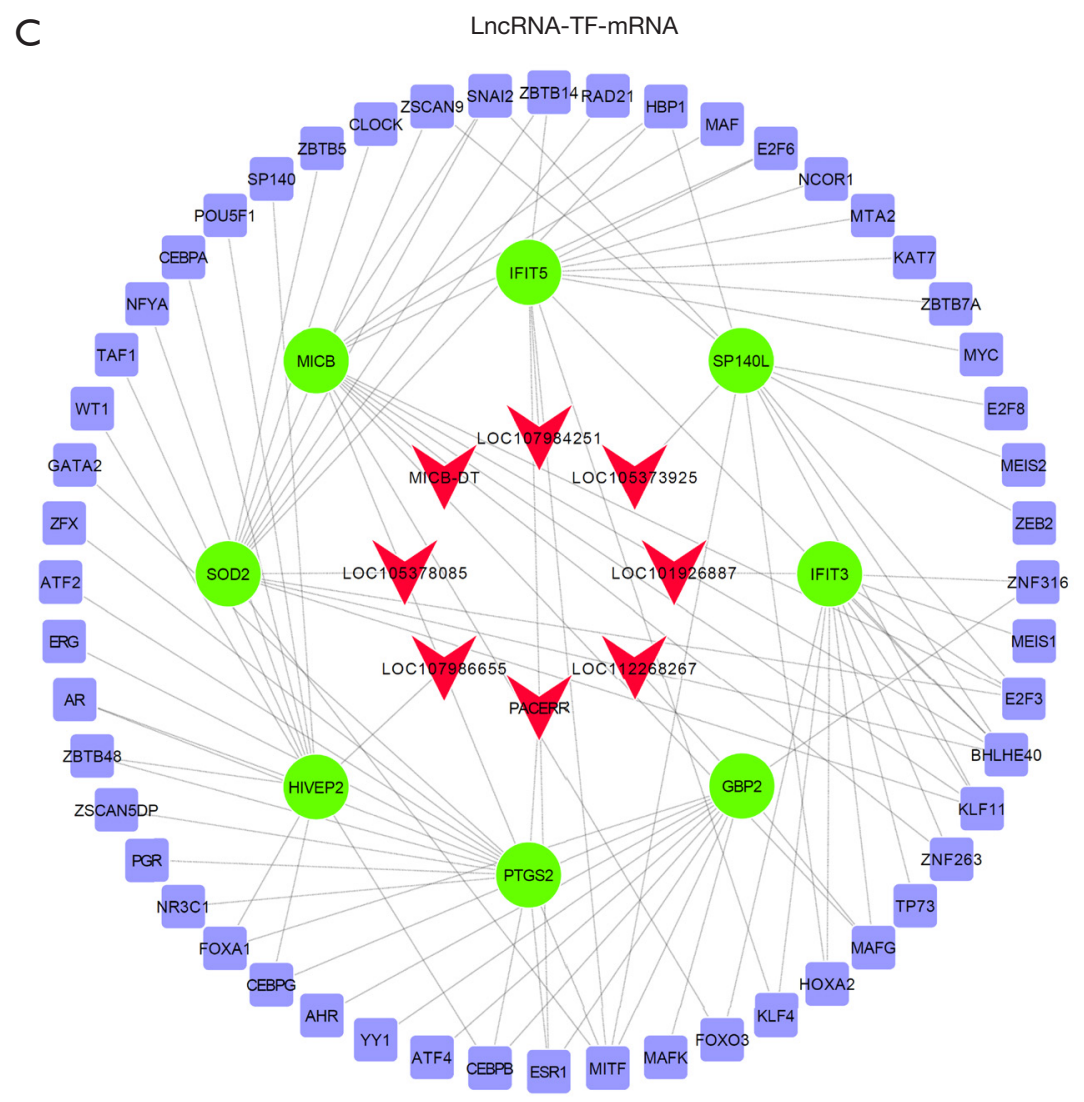

Figure 6 LncRNA-TF-mRNA network. (A) Top 20 terms for cis-regulation prediction. (B) Predicted trans-regulation of DE lncRNAs and mRNAs. The red triangle and deep green ellipse represent lncRNAs and TFs, respectively. (C) LncRNA-TF-mRNA network. The red triangle, green ellipse, and blue rectangle represent lncRNAs, mRNAs, and TFs, respectively. * $\mathrm{P}<0.05$; **, $\mathrm{P}<0.01$. LncRNAs, long noncoding RNAs; TF, transcription factor; mRNAs, messenger RNAs; DE, differentially-expressed.

maintain the balance of Th17/Treg cells and the imbalance of Th17/Treg cell subsets may contribute to inflammatory responses, suggesting that the reduced immunoregulatory ability of AS-MSCs may play a key role in the pathogenesis of AS (8,32). Accordingly, MSCs have been used to exert considerable therapeutic effects on several autoimmune diseases owing to their multilineage differentiation potential and high immunoregulatory properties (33,34). Notably, we also attempted to infuse MSCs from HDs into AS patients intravenously, since the immunoregulatory function of AS-MSCs themselves was reduced. The results of our clinical trial demonstrated that infusion of MSCs isolated from healthy individuals is a safe and efficient method for the treatment of AS (7). Unfortunately, the cause of the abnormal immunoregulatory ability of AS-MSCs remains unclear and needs to be addressed.

Accumulating evidence suggests that activation of TLRs can modulate the immunoregulatory functions of MSCs (15-17). In addition, emerging evidence further suggests a role for TLRs in the pathogenesis of spondyloarthropathies, including AS (13). Although inconsistent results of TLR4 immunoregulation in MSCs have been reported in recent years (15-17). The potential causes of this polarizing effect could be attributed to the specific stimulation protocols and culture conditions used including experimental setups, TLR agonist concentrations and stimulation times, sources of MSCs as effector cells, and PBMC or purified T cells as responder cell, which adds to the complexity of the situation. According to our previous study, the expression of TLR4 was downregulated in MSCs derived from AS patients, and compared with MSCs from HDs, TLR4primed AS-MSCs possessed enhanced immunoregulatory effects limiting the proliferation of naive $\mathrm{CD}^{+} \mathrm{T}$ cells (19). TLR4 is essential for the suppressive activity of MSCs, as 
TLR4 deletion is associated with a diminished suppressive effect in vitro and a loss of therapeutic effect in MSCsTLR4 knockout mice. Additionally, the immunoplasticity of MSCs through TLR4 activation is time-dependent to LPS exposure, which can lead to opposite therapeutic outcome in EAE (18). However, the precise mechanism underlying the enhanced immunoregulatory ability of TLR4-primed AS-MSCs remains unclear. Therefore, we measured the differential expression profiles of lncRNAs and mRNAs in AS-MSCs after TLR4 activation to identify the regulatory network of lncRNAs and mRNAs in these cells. The results showed that there were $698 \mathrm{DE}$ mRNAs and 147 DE lncRNAs in TLR4-primed AS-MSCs compared with unstimulated cells. The top five mRNAs and lncRNAs, which may be involved in the regulatory dysfunction of ASMSCs, were verified by qRT-PCR.

The mRNA expression profiles reflect the biological behaviors and functions of cells. In this study, KEGG pathway analysis revealed that 75 signaling pathways exhibited significant differences between TLR4-primed ASMSCs and unstimulated AS-MSCs. Among these pathways, the NLR signaling pathway, TNF signaling pathway, $\mathrm{NF}-\kappa \mathrm{B}$ signaling pathway, cytokine-cytokine receptor interaction, and TLR signaling pathway were prominent. Recent studies have indicated that the activation of TLRs can activate the NF- $\kappa \mathrm{B}$ and MAPK signaling pathways to promote the secretion of pro-inflammatory cytokines, such as IL-6, IL-12, TNF- $\alpha$, and type I IFNs, which drive inflammation in AS (35). Consistent with these findings, we found that the expression of TNF- $\alpha$, IL- 6 , and IL- $1 \beta$ in AS-MSCs was significantly increased after TLR4 activation, which supported the crucial roles of TLR4 in the NF- $\mathrm{KB}$ and MAPK signaling pathways identified by microarray analysis. In addition, as pathogen recognition receptors, both TLR and NLR activate pathways mediated by different adaptor proteins that are commonly found to activate NF$\kappa \mathrm{B}(36)$. The NF- $\kappa \mathrm{B}$-mediated activation of MSCs leads to the secretion of TNF- $\alpha$ and other cytokines. Elevated pro-inflammatory cytokine levels are one of the main manifestations of AS, as confirmed by previous research. Our results further confirmed the significant role of TLR4 in the pathology of AS. The selected top DE mRNAs and

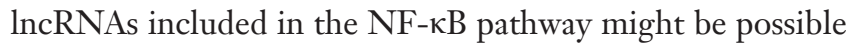
upstream targets of pathological inflammation in AS.

The mRNA expression profiles are under the control of a series of epigenetic regulators, of which lncRNAs are an important component $(22,37)$. In recent years, an increasing number of lncRNAs have been reported to perform key roles in the pathogenesis and development of AS. For example, $\operatorname{lnc} R N A-A K 001085$ expression was found to be downregulated in AS patients, which served as a potential diagnostic indicator; thus, this lncRNA is considered a potential suppressor of AS (38). Our previous microarray study identified four lncRNAs (lnc-ZNF354A-1, lncLIN 54-1, lnc-FRG2C-3, and lnc-USP50-2) that are involved in the abnormal osteogenic differentiation of AS-MSCs (39). However, the immunoregulatory function of AS-MSCs regulated by lncRNAs has not been explored. Our research identifies the lncRNA expression profile in an inflammatory environment based on previous studies, which provides a possible way to further explore the regulatory function of lncRNAs in AS. To the best of our knowledge, this study is the first to use microarray analyses to examine the roles of lncRNAs in TLR4-primed AS-MSCs.

In this study, several lncRNAs with the largest fold changes among DE lncRNAs were studied. For example, GBP1P1 is a lncRNA that acts as a prognostic biomarker for hepatocellular carcinoma (40). MIR3142HG can regulate the inflammatory response following IL- $1 \beta$-mediated activation of human lung fibroblasts, which is a positive regulator of IL-8 and CCL2 release (41). The inflammatory response regulation by MIR3142HG indicates that it may contribute to the enhanced immunoregulatory ability of TLR4-primed AS-MSCs and the immunoregulatory dysfunction seen in AS. Unfortunately, most of the DE lncRNAs, such as LOC101926887, LOC105378410 and LOC107984251, have not yet been studied; further exploration is needed in the future.

Regulatory lncRNAs act in a cis and/or trans manner to influence or interact with nearby or distant genes (42). In our study, eight lncRNA transcripts were predicted to cis-regulate nearby protein-coding genes. In addition, we predicted the functions of trans-regulatory lncRNAs using TFs that regulate protein-coding gene expression. Fifteen lncRNAs were identified to function in a transregulation manner. Subsequently, eight core pairs of lncRNAs and target mRNAs in the lncRNA-TFmRNA network were obtained: PACERR-PTGS2, LOC105378085-SOD2, LOC107986655-HIVEP2, MICBDT-MICB, LOC105373925-SP140L, LOC107984251IFIT5, LOC112268267-GBP2 and LOC101926887-IFIT3. Among these core pairs, PACERR (PTGS2 Antisense NFKB1 Complex-Mediated Expression Regulator RNA, also known as PACER) is a novel lncRNA that has been found to interact with $\mathrm{NF}-\kappa \mathrm{B}$ transcriptional regulators to promote the expression of prostaglandin-endoperoxide 
synthase 2 (PTGS2, cyclooxygenase-2, also known as COX2) (43). COX2 can produce prostaglandin E2 (PGE2) and amplify the Th17-mediated autoimmune process via the COX2-PGE2-EP2/EP4-NF-кB loop (44). Targeting COX2 activity with non-steroidal anti-inflammatory drugs (NSAIDs) or agents designed to specifically block COX2 activity have been approved for therapeutic use (45). Notably, NSAIDs are recommended as the first-line treatment for AS patients due to their high efficiency in reducing back pain and stiffness in patients with axial spondyloarthritis (46). The pair of PACERR and PTGS2 may play a key role in the immunoregulation in TLR4primed AS-MSCs, which maybe an important potential biomarker for diagnosis and target for drug therapy. SOD2 is a component of the antioxidant defence systems, which are crucial in defending cells against oxidative stress. SOD2overexpressing BM-MSCs have been shown to exhibit an enhanced therapeutic effect on brain injury in traumatic brain injury mice (47). Moreover, MSCs could enhance the expression of the SOD2 antioxidant gene to adapt to the oxidative environment and exert their therapeutic effect (48). Exposure to LPS induces oxidative stress in ASMSCs, and LOC105378085 may regulate the expression of SOD2 in AS-MSCs to defend cells against oxidative stress and to exert the immunoregulatory effects of the ASMSCs. Finally, the above mentioned 23 DE lncRNA and eight lncRNA-target mRNA pairs identified in the present study provide novel information for understanding the biological functions of lncRNAs in AS-MSCs; however, the underlying mechanisms of how "IncRNA-TF-target gene" networks affect TLR4-induced immunoregulation needs to be clarified.

Our study has several limitations that should be noted. Firstly, RNA-seq is an important method to screen possible lncRNAs and mRNAs associated with specific diseases; however, the results of big-data analyses may include false positives. Therefore, we performed qRT-PCR to further verify the differential expression. Secondly, we predicted lncRNA functions only indirectly using bioinformatics analysis and validated several DE lncRNAs. Further functional studies on the mechanism are warranted to clarify the roles of lncRNAs.

\section{Conclusions}

TLR4 activation can enhance the immunoregulatory ability of MSCs in AS. This study described the lncRNA and mRNA expression profiles and functional networks in TLR4-primed AS-MSCs. Eight core pairs of lncRNA and target mRNA in the lncRNA-TF-mRNA network were identified in TLR4-primed AS-MSCs. These results provide insight into the pathogenesis of immunoregulatory dysfunction in AS-MSCs, which may help to elucidate possible molecular mechanisms and therapeutic targets in $\mathrm{AS}$.

\section{Acknowledgments}

Funding: This work was supported by the Guangdong Natural Science Foundation (grant numbers: 2020A1515011388), the Sun Yat-sen Memorial Hospital Yat-sen Qihang Youth Project (No. YXQH202009), and the China Postdoctoral Science Foundation (2020TQ0376).

\section{Footnote}

Reporting Checklist: The authors have completed the MDAR reporting checklist. Available at https://dx.doi. org/10.21037/atm-21-5020

Data Sharing Statement: Available at https://dx.doi. org/10.21037/atm-21-5020

Conflicts of Interest: All authors have completed the ICMJE uniform disclosure form (available at https://dx.doi. org/10.21037/atm-21-5020). The authors have no conflicts of interest to declare.

Ethical Statement: The authors are accountable for all aspects of the work in ensuring that questions related to the accuracy or integrity of any part of the work are appropriately investigated and resolved. The study was conducted in accordance with the Declaration of Helsinki (as revised in 2013). The present study was approved by the ethics committee of Sun Yat-sen Memorial Hospital, Sun Yat-sen University (No. 2020-KY-083). In addition, informed consent was obtained from all patients.

Open Access Statement: This is an Open Access article distributed in accordance with the Creative Commons Attribution-NonCommercial-NoDerivs 4.0 International License (CC BY-NC-ND 4.0), which permits the noncommercial replication and distribution of the article with the strict proviso that no changes or edits are made and the original work is properly cited (including links to both the formal publication through the relevant DOI and the license). 


\section{Page 16 of 17}

See: https://creativecommons.org/licenses/by-nc-nd/4.0/.

\section{References}

1. Braun J, Sieper J. Ankylosing spondylitis. Lancet 2007;369:1379-90.

2. Le Blanc K, Mougiakakos D. Multipotent mesenchymal stromal cells and the innate immune system. Nat Rev Immunol 2012;12:383-96.

3. Ghannam S, Pène J, Moquet-Torcy G, et al. Mesenchymal stem cells inhibit human Th17 cell differentiation and function and induce a $\mathrm{T}$ regulatory cell phenotype. $\mathrm{J}$ Immunol 2010;185:302-12.

4. Pistoia V, Raffaghello L. Mesenchymal stromal cells and autoimmunity. Int Immunol 2017;29:49-58.

5. He Y, Xu LL, Feng FE, et al. Mesenchymal stem cell deficiency influences megakaryocytopoiesis through the TNFAIP3/NF- $\mathrm{kB} / \mathrm{SMAD}$ pathway in patients with immune thrombocytopenia. Br J Haematol 2018;180:395-411.

6. Geng L, Tang X, Zhou K, et al. MicroRNA-663 induces immune dysregulation by inhibiting TGF- $\beta 1$ production in bone marrow-derived mesenchymal stem cells in patients with systemic lupus erythematosus. Cell Mol Immunol 2019;16:260-74.

7. Wang P, Li Y, Huang L, et al. Effects and safety of allogenic mesenchymal stem cell intravenous infusion in active ankylosing spondylitis patients who failed NSAIDs: a 20-week clinical trial. Cell Transplant 2014;23:1293-303.

8. Wu Y, Ren M, Yang R, et al. Reduced immunomodulation potential of bone marrow-derived mesenchymal stem cells induced CCR4+CCR6+ Th/Treg cell subset imbalance in ankylosing spondylitis. Arthritis Res Ther 2011;13:R29.

9. Santegoets KC, van Bon L, van den Berg WB, et al. Toll-like receptors in rheumatic diseases: are we paying a high price for our defense against bugs? FEBS Lett 2011;585:3660-6.

10. Mogensen TH, Paludan SR. Reading the viral signature by Toll-like receptors and other pattern recognition receptors. J Mol Med (Berl) 2005;83:180-92.

11. Chen JQ, Szodoray P, Zeher M. Toll-like receptor pathways in autoimmune diseases. Clin Rev Allergy Immunol 2016;50:1-17.

12. Dowling JK, Mansell A. Toll-like receptors: the swiss army knife of immunity and vaccine development. Clin Transl Immunology 2016;5:e85.

13. Tan FK, Farheen K. The potential importance of Toll-like receptors in ankylosing spondylitis. Int J Clin Rheumtol

\section{Li et al. Expression profiles of RNAs in TLR4-primed MSCs of AS}

2011;6:649-54.

14. Delarosa O, Dalemans W, Lombardo E. Toll-like receptors as modulators of mesenchymal stem cells. Front Immunol 2012;3:182.

15. Liotta F, Angeli R, Cosmi L, et al. Toll-like receptors 3 and 4 are expressed by human bone marrow-derived mesenchymal stem cells and can inhibit their T-cell modulatory activity by impairing Notch signaling. Stem Cells 2008;26:279-89.

16. Waterman RS, Tomchuck SL, Henkle SL, et al. A new mesenchymal stem cell (MSC) paradigm: polarization into a pro-inflammatory MSC1 or an Immunosuppressive MSC2 phenotype. PLoS One 2010;5:e10088.

17. Opitz CA, Litzenburger UM, Lutz C, et al. Toll-like receptor engagement enhances the immunosuppressive properties of human bone marrow-derived mesenchymal stem cells by inducing indoleamine-2,3-dioxygenase-1 via interferon-beta and protein kinase R. Stem Cells 2009;27:909-19.

18. Kurte M, Vega-Letter AM, Luz-Crawford P, et al. Time-dependent LPS exposure commands MSC immunoplasticity through TLR4 activation leading to opposite therapeutic outcome in EAE. Stem Cell Res Ther 2020;11:416.

19. Li Y, Huang L, Cai Z, et al. A study of the immunoregulatory function of TLR3 and TLR4 on mesenchymal stem cells in ankylosing spondylitis. Stem Cells Dev 2019;28:1398-412.

20. Beermann J, Piccoli MT, Viereck J, et al. Non-coding RNAs in development and disease: background, mechanisms, and therapeutic approaches. Physiol Rev 2016;96:1297-325.

21. Ning S, Li X. Non-coding RNA resources. Adv Exp Med Biol 2018;1094:1-7.

22. Kopp F, Mendell JT. Functional classification and experimental dissection of long noncoding RNAs. Cell 2018;172:393-407.

23. Yu L, Qu H, Yu Y, et al. LncRNA-PCAT1 targeting miR-145-5p promotes TLR4-associated osteogenic differentiation of adipose-derived stem cells. J Cell Mol Med 2018;22:6134-47.

24. Zhang CC, Niu F. LncRNA NEAT1 promotes inflammatory response in sepsis-induced liver injury via the Let-7a/TLR4 axis. Int Immunopharmacol 2019;75:105731.

25. Du M, Yuan L, Tan X, et al. The LPS-inducible lncRNA Mirt2 is a negative regulator of inflammation. Nat Commun 2017;8:2049. 
26. Li J, Wang M, Song L, et al. LncRNA MALAT1 regulates inflammatory cytokine production in lipopolysaccharidestimulated human gingival fibroblasts through sponging miR-20a and activating TLR4 pathway. J Periodontal Res 2020;55:182-90.

27. Xu Z, Zhou X, Li H, et al. Identification of the key genes and long non-coding RNAs in ankylosing spondylitis using RNA sequencing. Int J Mol Med 2019;43:1179-92.

28. Aggarwal S, Pittenger MF. Human mesenchymal stem cells modulate allogeneic immune cell responses. Blood 2005;105:1815-22.

29. Galipeau J, Sensébé L. Mesenchymal stromal cells: clinical challenges and therapeutic opportunities. Cell Stem Cell 2018;22:824-33.

30. Ma S, Xie N, Li W, et al. Immunobiology of mesenchymal stem cells. Cell Death Differ 2014;21:216-25.

31. Shi Y, Wang Y, Li Q, et al. Immunoregulatory mechanisms of mesenchymal stem and stromal cells in inflammatory diseases. Nat Rev Nephrol 2018;14:493-507.

32. Annunziato F, Cosmi L, Santarlasci V, et al. Phenotypic and functional features of human Th17 cells. J Exp Med 2007;204:1849-61.

33. Zahari W, Hashim SN, Yusof MF, et al. Immunomodulatory effect of cytokines in the differentiation of mesenchymal stem cells: a review. Curr Stem Cell Res Ther 2017;12:197-206.

34. Castro-Manrreza ME, Montesinos JJ. Immunoregulation by mesenchymal stem cells: biological aspects and clinical applications. J Immunol Res 2015;2015:394917.

35. Kawasaki T, Kawai T. Toll-like receptor signaling pathways. Front Immunol 2014;5:461.

36. Prajapati B, Jena PK, Rajput P, et al. Understanding and modulating the Toll like Receptors (TLRs) and NOD like Receptors (NLRs) cross talk in type 2 diabetes. Curr Diabetes Rev 2014;10:190-200.

37. Gardini A, Shiekhattar R. The many faces of long noncoding RNAs. FEBS J 2015;282:1647-57.

38. Li X, Chai W, Zhang G, et al. Down-regulation of lncRNA-AK001085 and its influences on the diagnosis of

Cite this article as: Li YX, Liu T, Liang YW, Huang JJ, Huang JS, Liu XG, Cheng ZY, Lu SX, Li M, Huang L. Integrative analysis of long non-coding RNA and messenger RNA expression in toll-like receptor 4-primed mesenchymal stem cells of ankylosing spondylitis. Ann Transl Med 2021;9(20):1563. doi: 10.21037/atm-21-5020 ankylosing spondylitis. Med Sci Monit 2017;23:11-6.

39. Xie Z, Li J, Wang P, et al. Differential expression profiles of long noncoding RNA and mRNA of osteogenically differentiated mesenchymal stem cells in ankylosing spondylitis. J Rheumatol 2016;43:1523-31.

40. Sui J, Miao Y, Han J, et al. Systematic analyses of a novel lncRNA-associated signature as the prognostic biomarker for hepatocellular carcinoma. Cancer Med 2018;7:3240-56.

41. Hadjicharalambous MR, Roux BT, Feghali-Bostwick CA, et al. Long non-coding RNAs are central regulators of the IL-1 $\beta$-induced inflammatory response in normal and idiopathic pulmonary lung fibroblasts. Front Immunol 2018;9:2906.

42. Sun M, Kraus WL. From discovery to function: the expanding roles of long noncoding RNAs in physiology and disease. Endocr Rev 2015;36:25-64.

43. Krawczyk M, Emerson BM. p50-associated COX2 extragenic RNA (PACER) activates COX-2 gene expression by occluding repressive NF- $\kappa \mathrm{B}$ complexes. Elife 2014;3:e01776.

44. Lee J, Aoki T, Thumkeo D, et al. T cell-intrinsic prostaglandin E2-EP2/EP4 signaling is critical in pathogenic TH17 cell-driven inflammation. J Allergy Clin Immunol 2019;143:631-43.

45. Salinas G, Rangasetty UC, Uretsky BF, et al. The cycloxygenase 2 (COX-2) story: it's time to explain, not inflame. J Cardiovasc Pharmacol Ther 2007;12:98-111.

46. Sieper J, Poddubnyy D. Axial spondyloarthritis. Lancet 2017;390:73-84.

47. Shi X, Bai Y, Zhang G, et al. Effects of over-expression of SOD2 in bone marrow-derived mesenchymal stem cells on traumatic brain injury. Cell Tissue Res 2018;372:67-75.

48. Fonteneau G, Bony C, Goulabchand R, et al. Serummediated oxidative stress from systemic sclerosis patients affects mesenchymal stem cell function. Front Immunol 2017;8:988.

(English Language Editor: A. Kassem) 
Supplementary

Table S1 Primers of the analyzed genes

\begin{tabular}{lll}
\hline Gene name & Forward primer & Reverse primer \\
\hline GAPDH & AAGGTGAAGGTCGGAGTCAA & AATGAAGGGGTCATTGATGG \\
MIR3142HG & TGGCTCAGAACTCCGATT & CCTACTCCTCACAGATACAC \\
LOC105374444 & AGCCCTCTTTGGTCAACTCC & TGGTTGCAGCATTGTCTTGG \\
PACERR & ATGTCTGTAGCATGCGGCTT & TTCAGAAAACAGGCCCCG \\
LOC105375914 & CCGTGTCTGGTCTGTACGTC & AACCTTACTCGCCCCAGTCT \\
CXCL1 & ACGCAAGCTGGGTTATTGGA & AGCAACTGTGGTTGTCAGAT \\
CXCL8 & CTCTTCTCCCTAGGAGCGTC & GATGCAGGATTGAGGCAAGC \\
CXCL10 & ACTGAGAGTGATTGAGAGTGGAC & AACCCTCTGCACCCAGTTTC \\
CXCL11 & GTGGCATTCAAGGAGTACCTC & TGATGGCCTTCGATTCTGGATT \\
CCL20 & GACGCTGTCTTTGCATAGGC & GGATTAGGCATCGTTGTCCTT \\
TNF- $\alpha$ & TGCTGTACCAAGAGTTGCTC & CGCACACAGACAACTTTTCTTT \\
CXCL9 & CCTCTCTCTAATCAGCCCTCTG & GAGGACCTGGGAGTAGATGAG \\
PDL1 & TGAGAAAGGTCGCTGTTCC & GGGCTTGGGGCAAATGTT \\
IL-1 $\beta$ & GCTGCACTAATTGTCTATTGGGA & AATTCGCTTGTAGTCGGCACC \\
LL-6 & ATGATGGCTTATTACAGTGGCAA & GTCGGAGATTCGTAGCTGGA \\
INOS & ACTCACCTCTTCAGAACGAATTG & CCATCTTTGGAAGGTTCAGGTTG \\
\hline
\end{tabular}

(c) Annals of Translational Medicine. All rights reserved. 
Table S2 GO analysis of DE mRNA

\begin{tabular}{|c|c|c|c|c|c|}
\hline ID & Term & Category & Count & Fold enrichment & $P$ value \\
\hline GO:0060337 & Type I interferon signaling pathway & Biological process & 26 & 13.95 & $1.30 \mathrm{E}-23$ \\
\hline GO:0060333 & Interferon-gamma-mediated signaling pathway & Biological process & 26 & 12.58 & $3.15 \mathrm{E}-22$ \\
\hline GO:0006954 & Inflammatory response & Biological process & 52 & 4.81 & 2.45E-21 \\
\hline GO:0045071 & Negative regulation of viral genome replication & Biological process & 16 & 14.09 & $4.25 \mathrm{E}-15$ \\
\hline GO:0051092 & Positive regulation of NF- $\kappa B$ TF activity & Biological process & 26 & 6.77 & 8.34E-15 \\
\hline GO:0006955 & Immune response & Biological process & 37 & 3.73 & $5.49 \mathrm{E}-12$ \\
\hline GO:0032496 & Response to LPS & Biological process & 24 & 5.12 & $4.75 \mathrm{E}-11$ \\
\hline GO:0045236 & CXCR chemokine receptor binding & Molecular function & 5 & 18.73 & 2.57E-06 \\
\hline GO:0004842 & Ubiquitin-protein transferase activity & Molecular function & 26 & 2.74 & 3.57E-06 \\
\hline GO:0003725 & Double-stranded RNA binding & Molecular function & 10 & 5.72 & $8.18 \mathrm{E}-06$ \\
\hline GO:0003700 & TF activity, sequence-specific DNA binding & Molecular function & 47 & 1.85 & 3.69E-05 \\
\hline GO:0016874 & Ligase activity & Molecular function & 19 & 2.81 & 5.17E-05 \\
\hline GO:0005125 & Cytokine activity & Molecular function & 16 & 3.10 & $6.26 \mathrm{E}-05$ \\
\hline GO:0004871 & Signal transducer activity & Molecular function & 17 & 2.95 & 6.83E-05 \\
\hline GO:0008270 & Zinc ion binding & Molecular function & 57 & 1.69 & 7.09E-05 \\
\hline GO:0005576 & Extracellular region & Cellular component & 66 & 1.61 & 7.97E-05 \\
\hline GO:0043657 & Host cell & Cellular component & 3 & 21.58 & 2.05E-04 \\
\hline GO:0033256 & $\mathrm{I}-\kappa \mathrm{B} / \mathrm{NF}-\kappa \mathrm{B}$ complex & Cellular component & 3 & 21.58 & 2.05E-04 \\
\hline GO:0097342 & Ripoptosome & Cellular component & 3 & 15.41 & $6.88 \mathrm{E}-04$ \\
\hline GO:0031264 & Death-inducing signaling complex & Cellular component & 3 & 15.41 & $6.88 \mathrm{E}-04$ \\
\hline GO:0000932 & Cytoplasmic mRNA processing body & Cellular component & 8 & 3.74 & $1.32 \mathrm{E}-03$ \\
\hline
\end{tabular}

GO, gene ontology; DE, differentially-expressed; mRNAs, messenger RNAs; TF, transcription factor; LPS, lipopolysaccharide. 
Table S3 Pathways with the largest significant difference of mRNA in KEGG analysis

\begin{tabular}{|c|c|c|c|c|c|}
\hline ID & Pathway & Count & $\begin{array}{l}\text { Fold } \\
\text { enrichment }\end{array}$ & $P$ value & Gene \\
\hline hsa04621 & $\begin{array}{l}\text { NLR signaling } \\
\text { pathway }\end{array}$ & 38 & 6.67 & 1.39E-21 & $\begin{array}{l}\text { CXCL8, GBP3, NAMPT, RBCK1, CXCL2, GBP4, OAS1, MAPK8, } \\
\text { GBP2, GBP1, TICAM1, AIM2, GBP5, IFNAR2, TNFAIP3, BIRC3, } \\
\text { IL1B, CXCL1, RIPK2, BIRC2, IRF9, MYD88, NLRP3, OAS2, IL6, } \\
\text { RIPK1, NFKB1, PANX1, CCL2, CCL5, IFNB1, CXCL3, ANTXR2, } \\
\text { STAT2, STAT1, CASP1, NOD1, OAS3 }\end{array}$ \\
\hline hsa05164 & Influenza A & 35 & 5.97 & $2.95 \mathrm{E}-18$ & $\begin{array}{l}\text { CXCL8, FAS, ICAM1, TLR3, RSAD2, OAS1, TNFSF10, CIITA, } \\
\text { MAPK8, HLA-DOB, TRIM25, MX1, TICAM1, IFNAR2, PML, } \\
\text { DDX58, IL1B, EIF2AK2, IRF9, MYD88, NLRP3, OAS2, IL6, } \\
\text { NFKB1, CCL2, CCL5, JAK2, IFNB1, CXCL10, IFNGR2, STAT2, } \\
\text { CASP1, STAT1, OAS3, IFIH1 }\end{array}$ \\
\hline hsa05168 & $\begin{array}{l}\text { Herpes simplex } \\
\text { infection }\end{array}$ & 34 & 5.42 & $2.12 \mathrm{E}-16$ & $\begin{array}{l}\text { HLA-F, FAS, SP100, TLR3, OAS1, MAPK8, HLA-DOB, CD74, } \\
\text { TICAM1, IFNAR2, PML, TNFSF14, DDX58, IL1B, EIF2AK2, IRF9, } \\
\text { MYD88, TRAF1, IFIT1, OAS2, NFKB1, IL6, CCL2, CCL5, JAK2, } \\
\text { IFNB1, TAP1, TAP2, IFNGR2, STAT2, C3, STAT1, OAS3, IFIH1 }\end{array}$ \\
\hline hsa04064 & $\begin{array}{l}\text { NF-kappa B signaling } \\
\text { pathway }\end{array}$ & 22 & 6.83 & 4.16E-13 & $\begin{array}{l}\text { CXCL8, CXCL2, ICAM1, VCAM1, PTGS2, TRIM25, TICAM1, } \\
\text { TNFAIP3, TNFSF14, BIRC3, DDX58, IL1B, BIRC2, RELB, MYD88, } \\
\text { RIPK1, NFKB1, BCL2A1, CCL4, NFKB2, CFLAR, TRAF1 }\end{array}$ \\
\hline hsa04060 & $\begin{array}{l}\text { Cytokine-cytokine } \\
\text { receptor interaction }\end{array}$ & 36 & 3.93 & $8.02 \mathrm{E}-13$ & $\begin{array}{l}\text { CXCL8, IL6R, FLT3, CXCL9, CXCL2, FAS, CSF1, IL15RA, } \\
\text { TNFSF10, CXCL11, TNFSF9, IL1RAP, BMP2, IFNAR2, TNFSF14, } \\
\text { IL1B, CXCL1, IL18R1, CCL7, CCL20, LIF, CXCL16, CXCL6, } \\
\text { CX3CL1, IL6, IL7, CCL2, CCL5, IFNB1, TSLP, CXCL3, CXCL5, } \\
\text { CXCL10, CCL4, IFNGR2, PDGFC }\end{array}$ \\
\hline hsa05162 & Measles & 22 & 4.84 & $5.58 \mathrm{E}-10$ & $\begin{array}{l}\text { FAS, OAS1, TNFSF10, MX1, IFNAR2, TNFAIP3, DDX58, IL1B, } \\
\text { EIF2AK2, IRF9, MYD88, OAS2, IL6, NFKB1, JAK2, IFNB1, } \\
\text { IFNGR2, STAT5A, STAT2, STAT1, OAS3, IFIH1 }\end{array}$ \\
\hline hsa04620 & $\begin{array}{l}\text { TLR signaling } \\
\text { pathway }\end{array}$ & 18 & 5.11 & 9.27E-09 & $\begin{array}{l}\text { CXCL8, TICAM1, MAP3K8, IL6, RIPK1, NFKB1, IFNAR2, } \\
\text { CXCL11, CCL5, IFNB1, MAPK8, IL1B, CXCL9, CXCL10, CCL4, } \\
\text { STAT1, MYD88, TLR3 }\end{array}$ \\
\hline
\end{tabular}

mRNA, messenger RNA; KEGG, Kyoto Encyclopedia of Genes and Genomes; NLR, NOD-like receptor; TLR, Toll-like receptor. 